\title{
On Two-Temperature Problem for Harmonic Crystals
}

\author{
T. V. Dudnikova, ${ }^{1}$ A. I. Komech, ${ }^{2,3}$ and N. J. Mauser $^{2}$
}

Received November 24, 2002; accepted March 12, 2003

\begin{abstract}
We consider the dynamics of a harmonic crystal in $d$ dimensions with $n$ components, $d, n \geqslant 1$. The initial date is a random function with finite mean density of the energy which also satisfies a Rosenblatt- or Ibragimov-Linnik-type mixing condition. The random function is translation-invariant in $x_{1}, \ldots, x_{d-1}$ and converges to different translation-invariant processes as $x_{d} \rightarrow \pm \infty$, with the distributions $\mu_{ \pm}$. We study the distribution $\mu_{t}$ of the solution at time $t \in \mathbb{R}$. The main result is the convergence of $\mu_{t}$ to a Gaussian translation-invariant measure as $t \rightarrow \infty$. The proof is based on the long time asymptotics of the Green function and on Bernstein's "room-corridor" argument. The application to the case of the Gibbs measures $\mu_{ \pm}=g_{ \pm}$with two different temperatures $T_{ \pm}$is given. Limiting mean energy current density is $-\left(0, \ldots, 0, C\left(T_{+}-T_{-}\right)\right)$with some positive constant $C>0$ what corresponds to Second Law.
\end{abstract}

KEY WORDS: Harmonic crystal; random initial data; mixing condition; Gaussian measures; covariance matrices; characteristic functional.

\section{INTRODUCTION}

The paper concerns the problems of the convergence to equilibrium distribution and the heat conduction for harmonic crystals. We have started in refs. 7-10 the analysis of the long time convergence to the equilibrium distribution for partial differential equations of hyperbolic type in $\mathbb{R}^{d}$ and for the harmonic crystals. Here we continue the analysis and prove Second Law for the crystals: the energy current is directed from the hot temperature to the cold one.

\footnotetext{
${ }^{1}$ M. V. Keldysh Institute of Applied Mathematics RAS, Moscow 125047, Russia; e-mail: dudnik @elsite.ru,dudnik@mat.univie.ac.at

${ }^{2}$ Institut für Mathematik, Wien A-1090, Austria; e-mail: komech@mat.univie.ac.at, mauser @)courant.nyu.edu

${ }^{3}$ On leave from Department of Mechanics and Mathematics, Moscow State University, Moscow 119899, Russia.
} 
For one-dimensional chains of harmonic oscillators (with $d=1$ ) similar results have been established in refs. 1, 27, and in refs. 2, 11, 12, 14, $15,16,23$ for one-dimensional chains of anharmonic oscillators coupled to heat baths. We extend the results for harmonic crystals to all $d \geqslant 1$. The case $d>1$ appears very different from $d=1$ because of more complicated properties of oscillatory integrals. We combine here the methods from ref. 1 with new ideas. Namely, we develop our "cutoff" strategy from ref. 10 which more carefully exploits the mixing condition in Fourier space. This approach allows us to cover all $d \geqslant 1$.

We assume that the initial state $Y_{0}(x)$ of the crystal is a random element of the Hilbert space $\mathscr{H}_{\alpha}$ of real sequences, see Definition 2.1 later. The distribution of $Y_{0}(x)$ is a probability measure $\mu_{0}$ of mean zero satisfying conditions S1-S3 later. In particular, the distribution of $Y_{0}(x)$ converges to distinct translation-invariant measures $\mu_{ \pm}$as $x_{d} \rightarrow \pm \infty$. Given $t \in \mathbb{R}$, denote by $\mu_{t}$ the probability measure that gives the distribution of the solution $Y(x, t)$ to dynamical equations with the random initial state $Y_{0}$. We study the asymptotics of $\mu_{t}$ as $t \rightarrow \pm \infty$.

Our main result gives the (weak) convergence of the measures $\mu_{t}$ on the Hilbert space $\mathscr{H}_{\alpha}$ with $\alpha<-d / 2$ to a limit measure $\mu_{\infty}$

$$
\mu_{t} \rightarrow \mu_{\infty}, \quad t \rightarrow \infty
$$

which is a translation-invariant Gaussian measure on $\mathscr{H}_{\alpha}$. A similar convergence result holds for $t \rightarrow-\infty$ since our system is time-reversible. We construct generic examples of harmonic crystals and random initial data satisfying all assumptions imposed. The explicit formulas for the covariance of the measure $\mu_{\infty}$ are given in (2.15)-(2.24). We derive the expression for the limit mean energy current $j_{\infty}$. The ergodicity and mixing of the limit measures $\mu_{\infty}$ follow by the same arguments as in ref. 10 .

We apply our results to a particular case when $\mu_{ \pm}=g_{ \pm}$are Gibbs measures with two distinct temperatures $T_{ \pm} \geqslant 0$ (we adjust the definition of the Gibbs measures $g_{ \pm}$in Section 4 ). The measures $g_{ \pm}$satisfy all our assumptions, and the weak convergence $g_{t} \rightarrow g_{\infty}$ follows from our results. We apply formula for the limit energy current $j_{\infty} \equiv\left(j_{\infty}^{1}, \ldots, j_{\infty}^{d}\right)$ to the case of Gibbs measures $g_{ \pm}$and deduce that

$$
j_{\infty}=-\left(0, \ldots, 0, C\left(T_{+}-T_{-}\right)\right), \quad C>0 .
$$

This corresponds to Second Law.

For $d=1$ similar problem in different framework have been analyzed in refs. 20 and 24. The authors follow another strategy for the construction of equilibrium measure. Namely, the finite simple lattice of length $L$ with 
the viscosity is considered in contact with two heat baths at temperatures $T_{ \pm}$. The convergence of the covariance is proved in the limits first $t \rightarrow \infty$, and then $L \rightarrow \infty$. The result is close to ours: the limit energy current is nonzero and $\sim \Delta T$ which corresponds to the superconductivity. ${ }^{(2)}$ However, the space decay of the limit position-momentum covariance in ref. 20 is exponential which differs from the power decay in our problem (see Remark 4.2(iii)). Therefore, the equilibrium measures are distinct.

For $d \geqslant 1$ the convergence (1.1) has been obtained for the first time in ref. 19 for initial measures which are absolutely continuous with respect to the canonical Gaussian measure. We cover more general class of initial measures with the mixing condition and do not assume the absolute continuity. For the first time the mixing condition has been introduced by R. Dobrushin and Yu. Suhov for the ideal gas. ${ }^{(4)}$ The condition substitutes (quasi-) ergodic hypothesis in the proof of the convergence to the equilibrium distribution, and plays the key role in our Bernstein-type approach. Developing this approach, we have proved the convergence for the wave and Klein-Gordon equations with translation-invariant initial measures. ${ }^{(7,8,18)}$ In ref. 9 we have extended the results to the wave equation with the two-temperature initial measures. The present paper develops our previous results, ${ }^{(10)}$ where the harmonic crystal has been considered for all $d \geqslant 1$ in the case of translation invariant initial measures. Here we extend the results to the two-temperature initial measures.

We outline our main result and strategy of proof. Consider a discrete subgroup $\Gamma$ of $\mathbb{R}^{d}$, which is isomorphic to $\mathbb{Z}^{d}$. We may assume $\Gamma=\mathbb{Z}^{d}$ after a suitable change of coordinates. A lattice in $\mathbb{R}^{d}$ is the set of the points of the form $\bar{r}_{\lambda}(x)=x+\xi_{\lambda}$, where $x \in \mathbb{Z}^{d}, \xi_{\lambda} \in \mathbb{R}^{d}, \lambda=1, \ldots, \Lambda$. The points of the lattice represent the equilibrium positions of the atoms (molecules, ions,...) of the crystal. Denote by $r_{\lambda}(x, t)$ the positions of the atoms in the dynamics. Then the dynamics of the displacements $r_{\lambda}(x, t)-\bar{r}_{\lambda}(x)$ is governed by the equations of type

$$
\left\{\begin{array}{l}
\ddot{u}(x, t)=-\sum_{y \in \mathbb{Z}^{d}} V(x-y) u(y, t), \quad x \in \mathbb{Z}^{d}, \\
\left.u\right|_{t=0}=u_{0}(x),\left.\quad \dot{u}\right|_{t=0}=v_{0}(x) .
\end{array}\right.
$$

Here $u(x, t)=\left(u_{1}(x, t), \ldots, u_{n}(x, t)\right), u_{0}=\left(u_{01}, \ldots, u_{0 n}\right), v_{0}=\left(v_{01}, \ldots, v_{0 n}\right) \in \mathbb{R}^{n}$, $n=\Lambda d ; V(x)$ is the real interaction (or force) matrix, $\left(V_{k l}(x)\right), k, l=1, \ldots, n$. Similar equations were considered in refs. $1,10,19$, and 27 . Below we consider the system of type (1.2) with an arbitrary $n=1,2, \ldots$.

Denote $Y(t)=\left(Y^{0}(t), Y^{1}(t)\right) \equiv(u(\cdot, t), \dot{u}(\cdot, t)), Y_{0}=\left(Y_{0}^{0}, Y_{0}^{1}\right) \equiv\left(u_{0}(\cdot)\right.$, $\left.v_{0}(\cdot)\right)$. Then (1.2) takes the form of an evolution equation

$$
\dot{Y}(t)=\mathscr{A} Y(t), \quad t \in \mathbb{R} ; \quad Y(0)=Y_{0} .
$$


Formally, this is the Hamiltonian system since

$$
\mathscr{A} Y=J\left(\begin{array}{ll}
\mathscr{V} & 0 \\
0 & 1
\end{array}\right) Y=J \nabla H(Y), \quad J=\left(\begin{array}{cc}
0 & 1 \\
-1 & 0
\end{array}\right) .
$$

Here $\mathscr{V}$ is a convolution operator with the matrix kernel $V$ and $H$ is the Hamiltonian functional

$$
H(Y):=\frac{1}{2}\langle v, v\rangle+\frac{1}{2}\langle\mathscr{V} u, u\rangle, \quad Y=(u, v),
$$

where the kinetic energy is given by $\frac{1}{2}\langle v, v\rangle=\frac{1}{2} \sum_{x \in \mathbb{Z}^{d}}|v(x)|^{2}$ and the potential energy by $\frac{1}{2}\langle\mathscr{V} u, u\rangle=\frac{1}{2} \sum_{x, y \in \mathbb{Z}^{d}}(V(x-y) u(y), u(x)), \quad(\cdot, \cdot)$ stands for the real scalar product in the Euclidean space $\mathbb{R}^{n}$.

We assume that the initial correlation functions

$$
Q_{0}^{i j}(x, y):=E\left(Y_{0}^{i}(x) \otimes Y_{0}^{j}(y)\right), \quad x, y \in \mathbb{Z}^{d},
$$

have the form

$$
Q_{0}^{i j}(x, y)=q_{0}^{i j}\left(\bar{x}-\bar{y}, x_{d}, y_{d}\right), \quad i, j=0,1 .
$$

Here $x=\left(x_{1}, \ldots, x_{d}\right) \equiv\left(\bar{x}, x_{d}\right), y=\left(y_{1}, \ldots, y_{d}\right) \equiv\left(\bar{y}, y_{d}\right) \in \mathbb{Z}^{d}$. Moreover, we assume that

$$
\lim _{y_{d} \rightarrow \pm \infty} q_{0}^{i j}\left(\bar{z}, y_{d}+z_{d}, y_{d}\right)=q_{ \pm}^{i j}(z), \quad z=\left(\bar{z}, z_{d}\right) \in \mathbb{Z}^{d} .
$$

Here $q_{ \pm}^{i j}(z)$ are the correlation functions of some translation-invariant measures $\mu_{ \pm}$with zero mean value in $\mathscr{H}_{\alpha}$. The measure $\mu_{0}$ is not translationinvariant if $q_{-}^{i j} \neq q_{+}^{i j}$.

Next, we assume that the initial mean "energy" density is uniformly bounded:

$$
\begin{aligned}
e_{0}(x) & :=E\left[\left|u_{0}(x)\right|^{2}+\left|v_{0}(x)\right|^{2}\right] \\
& =\operatorname{tr} Q_{0}^{00}(x, x)+\operatorname{tr} Q_{0}^{11}(x, x) \leqslant e_{0}<\infty, \quad x \in \mathbb{Z}^{d} .
\end{aligned}
$$

Finally, it is assumed that the measure $\mu_{0}$ satisfies a mixing condition of a Rosenblatt- or Ibragimov-Linnik-type, which means that

$Y_{0}(x)$ and $Y_{0}(y)$ are asymptotically independent as $|x-y| \rightarrow \infty$.

To prove the convergence (1.1) we follow the strategy of refs. 7-10. There are three steps: 
(I) The family of measures $\mu_{t}, t \geqslant 0$, is weakly compact in $\mathscr{H}_{\alpha}$, $\alpha<-d / 2$.

(II) The correlation functions converge to a limit,

$$
Q_{t}^{i j}(x, y) \equiv \int\left(Y^{i}(x) \otimes Y^{j}(y)\right) \mu_{t}(d Y) \rightarrow Q_{\infty}^{i j}(x, y), \quad t \rightarrow \infty
$$

(III) The characteristic functionals converge to a Gaussian one,

$$
\hat{\mu}_{t}(\Psi):=\int e^{i\langle Y, \Psi\rangle} \mu_{t}(d Y) \rightarrow \exp \left\{-\frac{1}{2} \mathscr{Q}_{\infty}(\Psi, \Psi)\right\}, \quad t \rightarrow \infty
$$

Here $\Psi=\left(\Psi^{0}, \Psi^{1}\right) \in \mathscr{D}:=D \oplus D, D:=C_{0}\left(\mathbb{Z}^{d}\right) \otimes \mathbb{R}^{n}$, where $C_{0}\left(\mathbb{Z}^{d}\right)$ denotes a space of real sequences with finite support, $\langle Y, \Psi\rangle=\sum_{i=0,1} \sum_{x \in \mathbb{Z}^{d}}$ $\left(Y^{i}(x), \Psi^{i}(x)\right)$, and $\mathscr{Q}_{\infty}$ is the quadratic form with the matrix kernel $\left(Q_{\infty}^{i j}(x, y)\right)_{i, j=0,1}$,

$$
\mathscr{Q}_{\infty}(\Psi, \Psi)=\sum_{i, j=0,1} \sum_{x, y \in \mathbb{Z}^{d}}\left(Q_{\infty}^{i j}(x, y), \Psi^{i}(x) \otimes \Psi^{j}(y)\right) .
$$

Below the brackets $\langle\cdot, \cdot\rangle$ denote also the Hermitian scalar product in the Hilbert spaces $L^{2}\left(T^{d}\right) \otimes \mathbb{R}^{n}$ or its different extensions.

For the proof of (I)-(III) we develop our cutting strategy from ref. 10 combined with some techniques from ref. 1 . To prove (II) we split $Q_{t}^{i j}(x, y)$ into even, odd components and the remainder as in ref. 1 . The even component corresponds to the translation-invariant initial measure and is analyzed by the method of ref. 10 for all $d \geqslant 1$. On the other hand, the odd component is missing in ref. 10 and it requires a novel idea since its Fourier transform contains the Cauchy Principal Value which is more singular than measures corresponding to the even component. The singularity was studied in ref. 1 for the case $d=1$. However, similar detailed analysis for $d>1$ seems to be impossible due to the bifurcations of the critical points.

Let us outline our method. We rewrite (1.11) in the equivalent form

$$
\mathscr{Q}_{t}(\Psi, \Psi) \rightarrow \mathscr{Q}_{\infty}(\Psi, \Psi), \quad t \rightarrow \infty,
$$

for $\Psi \in \mathscr{D}^{0}$ : by definition of $\mathscr{D}^{0}$, the Fourier transform $\hat{\Psi}(\theta)$ vanishes in a neighborhood of a "critical set" $\mathscr{C} \subset T^{d}$. The set $\mathscr{C}$ includes all points $\theta \in T^{d}$ with a degenerate Hessian of $\omega_{k}(\theta)$, where $\omega_{k}^{2}(\theta)$ are the eigenvalues of the matrix $\hat{V}(\theta)=\sum_{z \in \mathbb{Z}^{d}} e^{i z \theta} V(z)$. Also the set $\mathscr{C}$ includes the points $\theta \in T^{d}$ either with $\omega_{k}(\theta)=0$, or $\nabla_{\theta_{d}} \omega_{k}(\theta)=0$ or with non-smooth $\omega_{k}(\theta)$. The cutting of the critical set $\mathscr{C}$ is possible by two key observations: 
(i) mes $\mathscr{C}=0$ and (ii) the correlation quadratic form is continuous in $l^{2}$ due to the mixing condition. The continuity follows from the space decay of correlation functions by well-known Shur's lemma. The systematic application of the Shur lemma allows us to extend (1.14) from $\Psi \in \mathscr{D}^{0}$ to all $\Psi \in \mathscr{D}$ by condition E6.

Similarly, we first prove the property (III) for $\Psi \in \mathscr{D}^{0}$ and then extend it to all $\Psi \in \mathscr{D}$. For $\Psi \in \mathscr{D}^{0}$ we use a variant of the S. N. Bernstein "roomcorridor" technique (cf. ref. 1 for $d=1$ ). We develop our variant of the S. N. Bernstein technique which we have introduced in refs. 7-9, 18 in the context of the Klein-Gordon and wave equations and in ref. 10 for the harmonic crystal with $d \geqslant 1$ in the case of translation-invariant initial measures. For $\Psi \in \mathscr{D}^{0}$ we have $\hat{\mu}_{t}(\Psi)=E \exp (i\langle Y(t), \Psi\rangle)$. We rewrite, $\langle Y(t), \Psi\rangle=\langle Y(0), \Phi(\cdot, t)\rangle$, where $\Phi(x, t)$ and be represented as an oscillatory integral. For $\Phi(x, t)$ we get the uniform bounds (9.6), (9.7). These bounds follow by the stationary phase method because $\Phi(x, 0)=$ $\Psi(x) \in \mathscr{D}^{0}$, and hence, $\hat{\Psi}(\theta)$ vanishes in all points $\theta \in \mathscr{C}$ with degenerate Hessian of the phase function. The bounds roughly speaking imply the following representation:

$$
\langle Y(\cdot, t), \Psi\rangle \sim \frac{\sum_{y \in B_{t}} Y_{0}(y)}{\sqrt{\left|B_{t}\right|}}, \quad t \rightarrow \infty,
$$

where $B_{t}$ stands for the ball $\left\{y \in \mathbb{Z}^{d}:|y| \leqslant c t\right\}$ and $\left|B_{t}\right|$ is its volume. Now (1.12) follows from (1.15) by the Lindeberg Central Limit Theorem since $Y_{0}\left(y_{1}\right), Y_{0}\left(y_{2}\right)$ are almost independent for large $\left|y_{1}-y_{2}\right|$ by mixing condition (1.10).

Let us comment on our conditions concerning the interaction matrix $V(x)$. We assume conditions E1-E4 later which in a similar form appear also in refs. 1, 19, and 26. E1 means the exponential space-decay of the interaction in the crystal. E2 resp. E3 means that the potential energy is real resp. nonnegative. We need condition E4 to apply the stationary phase method to the oscillatory integral representation for the covariance. It provides that the stationary points of the phase function are nondegenerate and ensures that mes $\mathscr{C}=0$. We also introduce a new simple condition E5 for the case $n>1$ which provides the convergence of the covariance $Q_{t}$. It can be considerably weakened to condition E5' from Remark 2.9(iii). For example, condition E5' holds for the canonical Gaussian measures which are considered in ref. 19. Conditions E4 and E5 hold for almost all functions $V(x)$ satisfying E1-E3 as shown in ref. 10. Furthermore, we do not require that $\omega_{k}(\theta) \neq 0$ : for instance, $\omega_{k}(0)=0$ for the elastic lattice (3.2) in the case $m_{k}=0$. Instead we require that $\operatorname{mes}\left\{\theta \in T^{d}: \omega_{k}(\theta)=0\right\}=0$ and 
impose condition E6 which is similar to condition (iii) from ref. 19, p. 171. E6 holds for the elastic lattice (3.2) if either $d \geqslant 3$ or $m_{k}>0$.

The main result of the paper is stated in Section 2 (see Theorem A). In Section 3 we give examples of Eq. (1.2) and measures $\mu_{0}$ which satisfy all our conditions E1-E6 and S0-S3, respectively. Section 4 concerns the application to Gibbs measures. In Section 5 we give bounds for the initial covariance. The compactness (Property I) is established in Section 6, convergence (1.11) in Sections 7 and 8, and convergence (1.12) in Section 9. In Section 10 we check the Lindeberg condition for convergence to a Gaussian limit. Appendix A is concerned with a dynamics and covariance in Fourier space.

\section{MAIN RESULTS}

Let us describe our results more precisely.

\subsection{Dynamics}

We assume that the initial date $Y_{0}$ belongs to the phase space $\mathscr{H}_{\alpha}$, $\alpha \in \mathbb{R}^{1}$, defined below.

Definition 2.1. $\quad \mathscr{H}_{\alpha}$ is the Hilbert space of pairs $Y \equiv(u(x), v(x))$ of $\mathbb{R}^{n}$-valued functions of $x \in \mathbb{Z}^{d}$ endowed with the norm

$$
\|Y\|_{\alpha}^{2} \equiv \sum_{x \in \mathbb{Z}^{d}}\left(|u(x)|^{2}+|v(x)|^{2}\right)\left(1+|x|^{2}\right)^{\alpha}<\infty
$$

We impose the following conditions E1-E6 on the matrix $V$.

E1. There exist constants $C, \alpha>0$ such that $\left|V_{k l}(z)\right| \leqslant C e^{-\alpha|z|}, k, l \in$ $\bar{n}:=\{1, \ldots, n\}, z \in \mathbb{Z}^{d}$.

Let us denote by $\hat{V}(\theta):=\left(\hat{V}_{k l}(\theta)\right)_{k, l \in \bar{n}}$, where $\hat{V}_{k l}(\theta) \equiv \sum_{z \in \mathbb{Z}^{d}} V_{k l}(z) e^{i z \theta}$, $\theta \in T^{d}$, and $T^{d}$ denotes the $d$-torus $\mathbb{R}^{d} / 2 \pi \mathbb{Z}^{d}$.

E2. $\quad V$ is real and symmetric, i.e., $V_{l k}(-z)=V_{k l}(z) \in \mathbb{R}, k, l \in \bar{n}, z \in \mathbb{Z}^{d}$.

The condition implies that $\hat{V}(\theta)$ is a real-analytic Hermitian matrixfunction in $\theta \in T^{d}$.

E3. The matrix $\hat{V}(\theta)$ is non-negative definite for each $\theta \in T^{d}$. 
The condition means that the Eq. (1.2) is hyperbolic like wave and Klein-Gordon equations considered in refs. 7 and 8 . Let us define the Hermitian non-negative definite matrix

$$
\Omega(\theta):=(\hat{V}(\theta))^{1 / 2} \geqslant 0
$$

with the eigenvalues $\omega_{k}(\theta) \geqslant 0, k \in \bar{n}$, which are called dispersion relations. For each $\theta \in T^{d}$ the Hermitian matrix $\Omega(\theta)$ has the diagonal form in the basis of the orthogonal eigenvectors $\left\{e_{k}(\theta): k \in \bar{n}\right\}$ :

$$
\Omega(\theta)=B(\theta)\left(\begin{array}{ccc}
\omega_{1}(\theta) & \cdots & 0 \\
0 & \ddots & 0 \\
0 & \cdots & \omega_{n}(\theta)
\end{array}\right) B^{*}(\theta),
$$

where $B(\theta)$ is a unitary matrix and $B^{*}(\theta)$ denotes its adjoint. It is well known that the functions $\omega_{k}(\theta)$ and $B(\theta)$ are real-analytic outside the set of the "crossing" points $\theta_{*}: \omega_{k}\left(\theta_{*}\right)=\omega_{l}\left(\theta_{*}\right)$ for some $l \neq k$. However, generally the functions are not smooth at the crossing points if $\omega_{k}(\theta) \not \equiv$ $\omega_{l}(\theta)$. Therefore, we need the following lemma which is proved in the Appendix of ref. 10 .

Lemma 2.2. Let conditions E1 and E2 hold. Then there exists a closed subset $\mathscr{C}_{*} \subset T^{d}$ such that (i) the Lebesgue measure of $\mathscr{C}_{*}$ is zero:

$$
\operatorname{mes} \mathscr{C}_{*}=0
$$

(ii) For any point $\Theta \in T^{d} \backslash \mathscr{C}_{*}$ there exists a neighborhood $\mathcal{O}(\Theta)$ such that each dispersion relation $\omega_{k}(\theta)$ and the matrix $B(\theta)$ can be chosen as the real-analytic functions in $\mathcal{O}(\Theta)$.

(iii) The eigenvalues $\omega_{k}(\theta)$ have constant multiplicity in $T^{d} \backslash \mathscr{C}_{*}$, i.e., it is possible to enumerate them so that we have for $\theta \in T^{d} \backslash \mathscr{C}_{*}$ :

$$
\begin{gathered}
\omega_{1}(\theta) \equiv \cdots \equiv \omega_{r_{1}}(\theta), \quad \omega_{r_{1}+1}(\theta) \equiv \cdots \equiv \omega_{r_{2}}(\theta), \ldots, \omega_{r_{s}+1}(\theta) \equiv \cdots \equiv \omega_{n}(\theta), \\
\omega_{r_{\sigma}}(\theta) \equiv \omega_{r_{v}}(\theta) \quad \text { if } \quad \sigma \neq v, \quad 1 \leqslant r_{\sigma}, \quad r_{v} \leqslant r_{s+1}:=n .
\end{gathered}
$$

(iv) The spectral decomposition holds,

$$
\Omega(\theta)=\sum_{\sigma=1}^{s+1} \omega_{r_{\sigma}}(\theta) \Pi_{\sigma}(\theta), \quad \theta \in T^{d} \backslash \mathscr{C}_{*},
$$


where $\Pi_{\sigma}(\theta)$ is the orthogonal projection in $\mathbb{R}^{n}$ which is real-analytic function of $\theta \in T^{d} \backslash \mathscr{C}_{*}$.

Below we suggest that $\omega_{k}(\theta)$ denote the local real-analytic functions from Lemma 2.2(ii). Our next condition is the following:

E4. $D_{k}(\theta) \not \equiv 0, \forall k \in \bar{n}$, where $D_{k}(\theta):=\operatorname{det}\left(\frac{\partial^{2} \omega_{k}(\theta)}{\partial \theta_{i} \partial \theta_{j}}\right)_{i, j=1}^{d}, \theta \in T^{d} \backslash \mathscr{C}_{*}$.

Let us denote $\mathscr{C}_{0}:=\left\{\theta \in T^{d}: \operatorname{det} \hat{V}(\theta)=0\right\}$ and $\mathscr{C}_{k}:=\left\{\theta \in T^{d} \backslash \mathscr{C}_{*}\right.$ : $\left.D_{k}(\theta)=0\right\}, k=1, \ldots, n$. The following lemma has also been proved in the Appendix of ref. 10.

Lemma 2.3. Let conditions E1-E4 hold. Then mes $\mathscr{C}_{k}=0, k=0$, $1, \ldots, n$.

Our last conditions on $V$ are the following:

E5. For each $k \neq l$ the identity $\omega_{k}(\theta)-\omega_{l}(\theta) \equiv$ const $_{-}, \theta \in T^{d}$ does not hold with const $\neq 00$, and the identity $\omega_{k}(\theta)+\omega_{l}(\theta) \equiv$ const $_{+}$does not hold with const $+\neq 0$.

E6. $\left\|\hat{V}^{-1}(\theta)\right\| \in L^{1}\left(T^{d}\right)$ in the case when $\mathscr{C}_{0} \neq \varnothing$.

This condition holds if $\mathscr{C}_{0}=\varnothing$.

The following Proposition 2.4 is proved in ref. 19, p. 150 and ref. 1, p. 128 (see also Appendix A).

Proposition 2.4. Let E1 and E2 hold, and $\alpha \in \mathbb{R}$. Then

(i) for any $Y_{0} \in \mathscr{H}_{\alpha}$ there exists a unique solution $Y(t) \in C\left(\mathbb{R}, \mathscr{H}_{\alpha}\right)$ to the Cauchy problem (1.3).

(ii) The operator $U(t): Y_{0} \mapsto Y(t)$ is continuous in $\mathscr{H}_{\alpha}$.

\subsection{Convergence to Statistical Equilibrium}

Let $(\Omega, \Sigma, P)$ be a probability space with expectation $E$ and $\mathscr{B}\left(\mathscr{H}_{\alpha}\right)$ denote the Borel $\sigma$-algebra in $\mathscr{H}_{\alpha}$. We assume that $Y_{0}=Y_{0}(\omega, \cdot)$ in $(1.3)$ is a measurable random function with values in $\left(\mathscr{H}_{\alpha}, \mathscr{B}\left(\mathscr{H}_{\alpha}\right)\right)$. In other words, for each $x \in \mathbb{Z}^{d}$ the map $\omega \mapsto Y_{0}(\omega, x)$ is a measurable map $\Omega \rightarrow \mathbb{R}^{2 n}$ with respect to the (completed) $\sigma$-algebras $\Sigma$ and $\mathscr{B}\left(\mathbb{R}^{2 n}\right)$. Then $Y(t)=U(t) Y_{0}$ is again a measurable random function with values in $\left.\left(\mathscr{H}_{\alpha}, \mathscr{B}_{(} \mathscr{H}_{\alpha}\right)\right)$ owing to Proposition 2.4. We denote by $\mu_{0}\left(d Y_{0}\right)$ a Borel probability measure on $\mathscr{H}_{\alpha}$ giving the distribution of the $Y_{0}$. Without loss of generality, we assume 
$(\Omega, \Sigma, P)=\left(\mathscr{H}_{\alpha}, \mathscr{B}\left(\mathscr{H}_{\alpha}\right), \mu_{0}\right)$ and $Y_{0}(\omega, x)=\omega(x)$ for $\mu_{0}(d \omega)$-almost all $\omega \in \mathscr{H}_{\alpha}$ and each $x \in \mathbb{Z}^{d}$.

Definition 2.5. $\mu_{t}$ is a Borel probability measure in $\mathscr{H}_{\alpha}$ which gives the distribution of $Y(t)$ :

$$
\mu_{t}(B)=\mu_{0}(U(-t) B), \quad \forall B \in \mathscr{B}\left(\mathscr{H}_{\alpha}\right), \quad t \in \mathbb{R} .
$$

Our main goal is to derive the convergence of the measures $\mu_{t}$ as $t \rightarrow \infty$. We establish the weak convergence of $\mu_{t}$ in the Hilbert spaces $\mathscr{H}_{\alpha}$ with $\alpha<-d / 2$ :

$$
\mu_{t} \stackrel{\mathscr{H}_{\alpha}}{\longrightarrow} \mu_{\infty} \quad \text { as } \quad t \rightarrow \infty,
$$

where $\mu_{\infty}$ is a limit measure on the space $\mathscr{H}_{\alpha}, \alpha<-d / 2$. This means the convergence

$$
\int f(Y) \mu_{t}(d Y) \rightarrow \int f(Y) \mu_{\infty}(d Y), \quad t \rightarrow \infty
$$

for any bounded continuous functional $f$ on $\mathscr{H}_{\alpha}$.

Definition 2.6. The correlation functions of the measure $\mu_{t}$ are defined by

$$
Q_{t}^{i j}(x, y)=E\left(Y^{i}(x, t) \otimes Y^{j}(y, t)\right), \quad i, j=0,1, \quad x, y \in \mathbb{Z}^{d},
$$

if the expectations in the r.h.s. are finite. Here $Y^{i}(x, t)$ are the components of the random solution $Y(t)=\left(Y^{0}(\cdot, t), Y^{1}(\cdot, t)\right)$.

For a probability measure $\mu$ on $\mathscr{H}_{\alpha}$ we denote by $\hat{\mu}$ the characteristic functional (Fourier transform)

$$
\hat{\mu}(\Psi) \equiv \int \exp (i\langle Y, \Psi\rangle) \mu(d Y), \quad \Psi \in \mathscr{D}
$$

A measure $\mu$ is called Gaussian (of zero mean) if its characteristic functional has the form

$$
\hat{\mu}(\Psi)=\exp \left\{-\frac{1}{2} \mathscr{L}(\Psi, \Psi)\right\}, \quad \Psi \in \mathscr{D},
$$

where $\mathscr{Q}$ is a real nonnegative quadratic form in $\mathscr{D}$. A measure $\mu$ is called translation-invariant if $\mu\left(T_{h} B\right)=\mu(B), B \in \mathscr{B}\left(\mathscr{H}_{\alpha}\right), h \in \mathbb{Z}^{d}$, where $T_{h} Y(x)=$ $Y(x-h), x \in \mathbb{Z}^{d}$. 


\subsection{Mixing Condition}

Let $O(r)$ denote the set of all pairs of subsets $\mathscr{A}, \mathscr{B} \subset \mathbb{Z}^{d}$ at distance $\operatorname{dist}(\mathscr{A}, \mathscr{B}) \geqslant r$ and let $\sigma(\mathscr{A})$ be a $\sigma$-algebra in $\mathscr{H}_{\alpha}$ generated by $Y(x)$ with $x \in \mathscr{A}$. Define the Ibragimov-Linnik mixing coefficient of a probability measure $\mu_{0}$ on $\mathscr{H}_{\alpha}$ by (cf. ref. 17, Definition 17.2.2)

$$
\varphi(r) \equiv \sup _{(\mathscr{A}, \mathscr{B}) \in O(r)} \sup _{\substack{A \in \sigma(\mathscr{A}), B \in \sigma(\mathscr{B}) \\ \mu_{0}(B)>0}} \frac{\left|\mu_{0}(A \cap B)-\mu_{0}(A) \mu_{0}(B)\right|}{\mu_{0}(B)} .
$$

Definition 2.7. The measure $\mu_{0}$ satisfies strong, uniform IbragimovLinnik mixing condition if

$$
\varphi(r) \rightarrow 0 \quad \text { as } \quad r \rightarrow \infty
$$

Below, we specify the rate of decay of $\varphi$ (see condition S3).

\subsection{Statistical Conditions and Results}

We assume that the initial measure $\mu_{0}$ satisfies the following conditions S0-S3:

So. $\mu_{0}$ has zero expectation value, $E Y_{0}(x)=0, x \in \mathbb{Z}^{d}$.

S1. $\mu_{0}$ has correlation functions of the form (1.7) with condition (1.8).

S2. $\mu_{0}$ has a finite mean energy density, i.e., Eq. (1.9) holds.

S3. $\mu_{0}$ satisfies the strong uniform Ibragimov-Linnik mixing condition with

$$
\bar{\varphi} \equiv \int_{0}^{+\infty} r^{d-1} \varphi^{1 / 2}(r) d r<\infty
$$

Introduce the correlation matrix of the limit measure $\mu_{\infty}$. It is translationinvariant

$$
Q_{\infty}(x, y)=\left(Q_{\infty}^{i j}(x, y)\right)_{i, j=0,1}=\left(q_{\infty}^{i j}(x-y)\right)_{i, j=0,1} .
$$


In the Fourier transform we have locally outside the critical set $\mathscr{C}_{*}$ (see Lemma 2.2)

$$
\hat{q}_{\infty}^{i j}(\theta)=B(\theta) M_{\infty}^{i j}(\theta) B^{*}(\theta), \quad i, j=0,1,
$$

where $B(\theta)$ is the smooth unitary matrix from Lemma 2.2(ii) and $M_{\infty}^{i j}(\theta)$ is $n \times n$-matrix with the smooth entries

$$
\begin{aligned}
M_{\infty}^{i j}(\theta)_{k l}= & \chi_{k l}\left[\left(B^{*}(\theta)\left(M_{0}^{+}\right)^{i j}(\theta) B(\theta)\right)_{k l}\right. \\
& \left.+i \operatorname{sgn}\left(\frac{\partial \omega_{k}}{\partial \theta_{d}}(\theta)\right)\left(B^{*}(\theta)\left(M_{0}^{-}\right)^{i j}(\theta) B(\theta)\right)_{k l}\right]
\end{aligned}
$$

Here we set (see (2.5))

$$
\chi_{k l}= \begin{cases}1 & \text { if } k, l \in\left(r_{\sigma-1}, r_{\sigma}\right], \quad \sigma=1, \ldots, s+1, \\ 0 & \text { otherwise }\end{cases}
$$

with $r_{0}:=0, r_{s+1}:=n$, and

$$
\begin{aligned}
& M_{0}^{+}(\theta):=\frac{1}{2}\left(\hat{\mathbf{q}}^{+}(\theta)+\hat{C}(\theta) \hat{\mathbf{q}}^{+}(\theta) \hat{C}^{*}(\theta)\right), \\
& M_{0}^{-}(\theta):=\frac{1}{2}\left(\hat{C}(\theta) \hat{\mathbf{q}}^{-}(\theta)-\hat{\mathbf{q}}^{-}(\theta) \hat{C}^{*}(\theta)\right),
\end{aligned}
$$

with $\mathbf{q}^{+}:=\frac{1}{2}\left(q_{+}+q_{-}\right), \mathbf{q}^{-}:=\frac{1}{2}\left(q_{+}-q_{-}\right)$and

$$
\hat{C}(\theta):=\left(\begin{array}{cc}
0 & \Omega^{-1}(\theta) \\
-\Omega(\theta) & 0
\end{array}\right), \quad \hat{C}^{*}(\theta):=\left(\begin{array}{cc}
0 & -\Omega(\theta) \\
\Omega^{-1}(\theta) & 0
\end{array}\right),
$$

where $\hat{C}^{*}$ denotes a Hermitian conjugate matrix to the matrix $\hat{C}$. The local representation (2.16) can be expressed globally as the sum:

$$
\hat{q}_{\infty}(\theta)=\hat{q}_{\infty}^{+}(\theta)+\hat{q}_{\infty}^{-}(\theta)
$$

where

$$
\begin{aligned}
&\left(\hat{q}_{\infty}^{+}\right)^{i j}(\theta):=\sum_{\sigma=1}^{s+1} \Pi_{\sigma}(\theta)\left(M_{0}^{+}\right)^{i j}(\theta) \Pi_{\sigma}(\theta) \\
&\left(\hat{q}_{\infty}^{-}\right)^{i j}(\theta):= \sum_{\sigma=1}^{s+1} i \operatorname{sgn}\left(\frac{\partial \omega_{r_{\sigma}}}{\partial \theta_{d}}(\theta)\right) \Pi_{\sigma}(\theta)\left(M_{0}^{-}\right)^{i j}(\theta) \Pi_{\sigma}(\theta), \\
& \theta \in T^{d} \backslash \mathscr{C}_{*}, \quad i, j=0,1 .
\end{aligned}
$$

Here $\Pi_{\sigma}(\theta)$ is the spectral projection introduced in Lemma 2.2(iv). 
Remark 2.8. From Proposition 5.2(ii) and condition E6 (if $\mathscr{C}_{0} \neq \varnothing$ ) it follows that $\left(\left(M_{0}^{ \pm}\right)^{i j}\right)_{k l} \in L^{1}\left(T^{d}\right), k, l \in \bar{n}$. Therefore, (2.23), (2.24), and (2.4) imply that also $\left(\left(\hat{q}_{\infty}^{ \pm}\right)^{i j}\right)_{k l} \in L^{1}\left(T^{d}\right), k, l \in \bar{n}$.

Theorem A. Let $d, n \geqslant 1, \alpha<-d / 2$, and assume that conditions E1-E5 and S0-S3 hold. If $\mathscr{C}_{0} \neq \varnothing$, then we assume also that E6 holds. Then

(i) the convergence (2.9) holds and (1.11) also holds.

(ii) The limit measure $\mu_{\infty}$ is a Gaussian translation-invariant measure on $\mathscr{H}_{\alpha}$.

(iii) The characteristic functional of $\mu_{\infty}$ is the Gaussian

$$
\hat{\mu}_{\infty}(\Psi)=\exp \left\{-\frac{1}{2} \mathscr{Q}_{\infty}(\Psi, \Psi)\right\}, \quad \Psi \in \mathscr{D},
$$

where $\mathscr{Q}_{\infty}$ is the quadratic form defined in (1.13).

(iv) The measure $\mu_{\infty}$ is invariant, i.e., $[U(t)]^{*} \mu_{\infty}=\mu_{\infty}, t \in \mathbb{R}$.

Remark 2.9. (i) In the case $d=n=1$ we have $B(\theta) \equiv 1$, and formulas (2.15)-(2.20) have been obtained in ref. 1, p. 139.

(ii) The uniform Rosenblatt mixing condition $^{(25)}$ also suffices, together with a higher power $>2$ in the bound (1.9): there exists $\delta>0$ such that

$$
E\left(\left|u_{0}(x)\right|^{2+\delta}+\left|v_{0}(x)\right|^{2+\delta}\right) \leqslant C<\infty .
$$

Then (2.14) requires a modification: $\int_{0}^{+\infty} r^{d-1} \alpha^{p}(r) d r<\infty$, where $p=$ $\min (\delta /(2+\delta), 1 / 2)$, where $\alpha(r)$ is the Rosenblatt mixing coefficient defined as in (2.12) but without $\mu_{0}(B)$ in the denominator. Under these modifications, the statements of Theorem $\mathrm{A}$ and their proofs remain essentially unchanged.

(iii) The arguments with condition E5 in Lemmas 8.1 and 8.2 demonstrate that the condition could be considerably weakened. Namely, it suffices to assume

E5'. If for some $k \neq l$ we have either $\omega_{k}(\theta)+\omega_{l}(\theta) \equiv$ const $_{+}$or $\omega_{k}(\theta)$ $-\omega_{l}(\theta) \equiv$ const $_{-}$with const ${ }_{ \pm} \neq 0$, then either $p_{k l}^{11}(\theta)-\omega_{k}(\theta) \omega_{l}(\theta) p_{k l}^{00}(\theta)$ $\equiv 0, \quad \omega_{k}(\theta) p_{k l}^{01}(\theta)+\omega_{l}(\theta) p_{k l}^{10}(\theta) \equiv 0, \quad$ or $\quad p_{k l}^{11}(\theta)+\omega_{k}(\theta) \omega_{l}(\theta) p_{k l}^{00}(\theta) \equiv 0$, $\omega_{k}(\theta) p_{k l}^{01}(\theta)-\omega_{l}(\theta) p_{k l}^{10}(\theta) \equiv 0$. Here

$$
p_{k l}^{i j}(\theta):=\left(B^{*}(\theta) \hat{q}_{ \pm}^{i j}(\theta) B(\theta)\right)_{k l}, \quad \theta \in T^{d}, \quad k, l \in \bar{n}, \quad i, j=0,1,
$$

$\hat{q}_{ \pm}^{i j}(\theta)$ are Fourier transforms of covariance matrices $q_{ \pm}^{i j}(z)$. 
Assertions (i)-(iii) of Theorem A follow from Propositions 2.10 and 2.11 below.

Proposition 2.10. The family of the measures $\left\{\mu_{t}, t \in \mathbb{R}\right\}$ is weakly compact in $\mathscr{H}_{\alpha}$ with any $\alpha<-d / 2$, and the bounds $\sup _{t \geqslant 0} E\left\|U(t) Y_{0}\right\|_{\alpha}^{2}$ $<\infty$ hold.

Proposition 2.11. For every $\Psi \in \mathscr{D}$ the convergence (1.12) holds.

Proposition 2.10 (Proposition 2.11) provides the existence (resp. the uniqueness) of the limit measure $\mu_{\infty}$. They are proved in Sections 6 and $8-10$, respectively.

Theorem A(iv) follows from (2.9) since the group $U(t)$ is continuous in $\mathscr{H}_{\alpha}$ by Proposition 2.4(ii).

\section{EXAMPLES}

Let us give the examples of Eq. (1.2) and measures $\mu_{0}$ which satisfy all our conditions E1-E6 and S0-S3, respectively.

\subsection{Nearest Neighbor Crystal}

Conditions E1-E6. For any $d, n \geqslant 1$ we consider the simple elastic lattice corresponding to the quadratic form

$$
\langle\mathscr{V} u, u\rangle=\sum_{k=1}^{n} \sum_{x \in \mathbb{Z}^{d}}\left(\sum_{i=1}^{d}\left|u^{k}\left(x+e_{i}\right)-u^{k}(x)\right|^{2}+m_{k}^{2}\left|u^{k}(x)\right|^{2}\right), \quad m_{k} \geqslant 0,
$$

where $e_{i}=\left(\delta_{i 1}, \ldots, \delta_{i d}\right)$. Then E1 holds and $\hat{V}(\theta)=\left(\omega_{k}^{2}(\theta) \delta_{k l}\right)_{k, l \in \bar{n}}$ with

$$
\omega_{k}(\theta)=\sqrt{2\left(1-\cos \theta_{1}\right)+\cdots+2\left(1-\cos \theta_{d}\right)+m_{k}^{2}}, \quad k \in \bar{n} .
$$

Hence, $V(x)$ satisfies E2-E4 with $\mathscr{C}_{*}=\varnothing$. By (3.2) the identities $\omega_{k}(\theta) \pm \omega_{l}(\theta) \equiv$ const $_{ \pm}$with const $_{ \pm} \neq 0$ are impossible, hence condition E5 holds. In the case when all $m_{k}>0$ the set $\mathscr{C}_{0}=\left\{\theta \in T^{d}: \operatorname{det} \hat{V}(\theta)=\right.$ $\left.\omega_{1}^{2}(\theta) \cdot \ldots \cdot \omega_{n}^{2}(\theta)=0\right\}$ is empty and condition E6 is unnecessary. Otherwise, if $m_{j}=0$ for some $j$, the set $\mathscr{C}_{0}=\{0\}$. Then E6 is equivalent to the condition $\omega_{j}^{-2}(\theta) \in L^{1}\left(T^{d}\right)$ that holds if $d \geqslant 3$.

Therefore, all conditions E1-E6 hold for (3.1) in the next cases: (i) $d \geqslant 3$, (ii) $d=1,2$, and all $m_{k}$ are positive.

Limit Covariance. For example, let us evaluate the limit covariance $q_{\infty}$ corresponding to (3.1) with $n=1$. Denote by $\mathscr{E}(z)=F_{\theta \rightarrow z}^{-1}\left(\omega^{-2}(\theta)\right)$ the 
fundamental solution for the operator $-\Delta+m^{2}$ on the lattice $\mathbb{Z}^{d}$, i.e., $\left(-\Delta+m^{2}\right) \mathscr{E}(x)=\delta_{0 x}$ for $x \in \mathbb{Z}^{d}$, and $P(x)=-i F_{\theta \rightarrow x}^{-1} \frac{\operatorname{sgn}\left(\sin \theta_{d}\right)}{\omega(\theta)}$. Then

$$
\begin{gathered}
q_{\infty}^{00}=\frac{1}{2}\left[\left(\mathbf{q}^{+}\right)^{00}+\mathscr{E} *\left(\mathbf{q}^{+}\right)^{11}+P *\left(\left(\mathbf{q}^{-}\right)^{01}-\left(\mathbf{q}^{-}\right)^{10}\right)\right], \\
q_{\infty}^{10}=-q_{\infty}^{01}=\frac{1}{2}\left[\left(\mathbf{q}^{+}\right)^{10}-\left(\mathbf{q}^{+}\right)^{01}+P *\left(\left(\mathbf{q}^{-}\right)^{11}+\left(-\Delta+m^{2}\right)\left(\mathbf{q}^{-}\right)^{00}\right)\right], \\
q_{\infty}^{11}=\left(-\Delta+m^{2}\right) q_{\infty}^{00}=\frac{1}{2}\left[\left(\mathbf{q}^{+}\right)^{11}+\left(-\Delta+m^{2}\right)\left(\left(\mathbf{q}^{+}\right)^{00}+P *\left(\left(\mathbf{q}^{-}\right)^{01}-\left(\mathbf{q}^{-}\right)^{10}\right)\right)\right],
\end{gathered}
$$

where $*$ stands for the convolution of functions.

\subsection{Gaussian Initial Measures}

For simplicity, we consider $n=1$ and construct Gaussian initial measures $\mu_{0}$ satisfying S0-S3. We will define $\mu_{ \pm}$in $\mathscr{H}_{\alpha}$ by the correlation functions $q_{ \pm}^{i j}(x-y)$ which are zero for $i \neq j$, while for $i=0,1$,

$$
\hat{q}_{ \pm}^{i i}(\theta):=F_{z \rightarrow \theta}\left[q_{ \pm}^{i i}(z)\right] \in L^{1}\left(T^{d}\right), \quad \hat{q}_{ \pm}^{i i}(\theta) \geqslant 0 .
$$

Then by the Minlos theorem, ${ }^{(3)}$ there exist Borel Gaussian measures $\mu_{ \pm}$ on $\mathscr{H}_{\alpha}, \alpha<-d / 2$, with the correlation functions $q_{ \pm}^{i j}(x-y)$, because formally we have

$$
\begin{aligned}
\int\|Y\|_{\alpha}^{2} \mu_{ \pm}(d Y) & =\sum_{x \in \mathbb{Z}^{d}}\left(1+|x|^{2}\right)^{\alpha}\left(\operatorname{tr} q_{ \pm}^{00}(0)+\operatorname{tr} q_{ \pm}^{11}(0)\right) \\
& =C(\alpha, d) \int_{T^{d}} \operatorname{tr}\left(\hat{q}_{ \pm}^{00}(\theta)+\hat{q}_{ \pm}^{11}(\theta)\right) d \theta<\infty
\end{aligned}
$$

The measures $\mu_{ \pm}$satisfy S0, S2. Let us take the functions $\zeta_{ \pm} \in C(\mathbb{Z})$ such that

$$
\zeta_{ \pm}(s)= \begin{cases}1, & \text { for } \pm s>a, \\ 0, & \text { for } \pm s<-a .\end{cases}
$$

Let us introduce $\left(Y_{-}, Y_{+}\right)$as a unit random function in probability space $\left(\mathscr{H}_{\alpha} \times \mathscr{H}_{\alpha}, \mu_{-} \times \mu_{+}\right)$. Then $Y_{ \pm}$are Gaussian independent vectors in $\mathscr{H}_{\alpha}$. Define a "two-temperature" Borel probability measure $\mu_{0}$ as a distribution of the random function

$$
Y_{0}(x)=\zeta_{-}\left(x_{d}\right) Y_{-}(x)+\zeta_{+}\left(x_{d}\right) Y_{+}(x) .
$$

Then correlation functions of $\mu_{0}$ are

$$
Q_{0}^{i j}(x, y)=q_{-}^{i j}(x-y) \zeta_{-}\left(x_{d}\right) \zeta_{-}\left(y_{d}\right)+q_{+}^{i j}(x-y) \zeta_{+}\left(x_{d}\right) \zeta_{+}\left(y_{d}\right), \quad i, j=0,1,
$$


where $x=\left(x_{1}, \ldots, x_{d}\right), y=\left(y_{1}, \ldots, y_{d}\right) \in \mathbb{Z}^{d}$, and $q_{ \pm}^{i j}$ are the correlation functions of the measures $\mu_{ \pm}$. The measure $\mu_{0}$ satisfies S0-S2. Further, let us assume, in addition to (3.3), that

$$
q_{ \pm}^{i i}(z)=0, \quad|z| \geqslant r_{0} .
$$

Then the mixing condition S3 follows with $\varphi(r)=0, r \geqslant r_{0}$. For instance, (3.3) and (3.7) hold if we set $q_{ \pm}^{i i}(z)=f\left(z_{1}\right) f\left(z_{2}\right) \cdots f\left(z_{d}\right)$, where $f(z)=$ $N_{0}-|z|$ for $|z| \leqslant N_{0}$ and $f(z)=0$ for $|z|>N_{0}$ with $N_{0}:=\left[r_{0} / \sqrt{d}\right]$ (the integer part). Then by the direct calculation we obtain $\hat{f}(\theta)=\left(1-\cos N_{0} \theta\right) /$ $(1-\cos \theta), \theta \in T^{1}$, and (3.3) holds.

\subsection{Non-Gaussian Initial Measures}

Let us choose some odd bounded nonconstant functions $f^{0}, f^{1} \in C(\mathbb{R})$. Define $\mu_{0}^{*}$ as the distribution of the random function $\left(f^{0}\left(Y^{0}(x)\right)\right.$, $\left.f^{1}\left(Y^{1}(x)\right)\right)$, where $\left(Y^{0}, Y^{1}\right)$ is a random function with a Gaussian distribution $\mu_{0}$ from the previous example. Then S0-S3 hold for $\mu_{0}^{*}$ with corresponding mixing coefficient $\varphi^{*}(r)=0$ for $r \geqslant r_{0}$. Measure $\mu_{0}^{*}$ is not Gaussian if the functions $f^{0}, f^{1}$ are bounded and nonconstant.

\section{APPLICATION TO SECOND LAW}

We apply Theorem A to the case when $\mu_{ \pm}$are the Gibbs measures corresponding to distinct positive temperatures $T_{-} \neq T_{+}$. We deduce that for the limit mean energy current $j_{\infty}=\left(j_{\infty}^{1}, \ldots, j_{\infty}^{d}\right)$ we have $j_{\infty}^{d}=$ $-C\left(T_{+}-T_{-}\right)$with $C>0$. Moreover, under the additional condition on $V$ we obtain $j_{\infty}^{k}=0, k=1, \ldots, d-1$. This means that the mean energy current is directed from high to low temperature in accordance with Second Law.

\subsection{Energy Current}

\subsubsection{Energy Current for Finite Energy Solutions}

We derive formally the expression for the energy current of the finite energy solutions $u(x, t)$ (see (1.5)). For the half-space $\Omega_{k}:=\left\{x \in \mathbb{Z}^{d}\right.$ : $\left.x_{k} \geqslant 0\right\}$ we define the energy in the region $\Omega_{k}(\mathrm{cf}(1.5))$ as

$$
\mathscr{E}_{k}(t):=\frac{1}{2} \sum_{x \in \Omega_{k}}\left\{|\dot{u}(x, t)|^{2}+\sum_{y \in \mathbb{Z}^{d}}(u(x, t), V(x-y) u(y, t))\right\} .
$$


By formal calculation, using Eq. (1.2) we obtain

$$
\begin{aligned}
\dot{\mathscr{E}}_{k}(t)= & \frac{1}{2}\left(\sum_{x \in \Omega_{k}^{c}, y \in \Omega_{k}}(\dot{u}(x, t), V(x-y) u(y, t))\right. \\
& \left.-\sum_{x \in \Omega_{k}, y \in \Omega_{k}^{c}}(\dot{u}(x, t), V(x-y) u(y, t))\right) .
\end{aligned}
$$

Here $\Omega_{k}^{c}:=\mathbb{Z}^{d} \backslash \Omega_{k}=\left\{x \in \mathbb{Z}^{d}: x_{k}<0\right\}$. Introduce new variables: $x=x^{\prime}+m e_{k}$, $y=y^{\prime}+p e_{k}$, where $x^{\prime}, y^{\prime} \in \mathbb{Z}^{d}$ with $x_{k}^{\prime}=y_{k}^{\prime}=0, e_{k}=\left(\delta_{k 1}, \ldots, \delta_{k d}\right), \quad k=$ $1, \ldots, d$. Then we rewrite (4.1) in the form

$$
\begin{aligned}
\dot{\mathscr{E}}_{k}(t)= & \frac{1}{2} \sum_{x^{\prime}, y^{\prime}}\left\{\sum_{m \leqslant-1, p \geqslant 0}\left(\dot{u}\left(x^{\prime}+m e_{k}, t\right), V\left(x^{\prime}+m e_{k}-y^{\prime}-p e_{k}\right) u\left(y^{\prime}+p e_{k}, t\right)\right)\right. \\
& \left.-\sum_{m \geqslant 0, p \leqslant-1}\left(\dot{u}\left(x^{\prime}+m e_{k}, t\right), V\left(x^{\prime}+m e_{k}-y^{\prime}-p e_{k}\right) u\left(y^{\prime}+p e_{k}, t\right)\right)\right\} \\
= & \sum_{x^{\prime}} j^{k}\left(x^{\prime}, t\right) .
\end{aligned}
$$

Here $j^{k}\left(x^{\prime}, t\right)$ stands for the energy current density in the direction $e_{k}$ : by definition,

$$
\begin{aligned}
j^{k}\left(x^{\prime}, t\right):= & \frac{1}{2} \sum_{y^{\prime}}\left\{\sum_{m \leqslant-1, p \geqslant 0}\left(\dot{u}\left(x^{\prime}+m e_{k}, t\right), V\left(x^{\prime}+m e_{k}-y^{\prime}-p e_{k}\right) u\left(y^{\prime}+p e_{k}, t\right)\right)\right. \\
& \left.-\sum_{m \geqslant 0, p \leqslant-1}\left(\dot{u}\left(x^{\prime}+m e_{k}, t\right), V\left(x^{\prime}+m e_{k}-y^{\prime}-p e_{k}\right) u\left(y^{\prime}+p e_{k}, t\right)\right)\right\}
\end{aligned}
$$

where $x^{\prime}, y^{\prime} \in \mathbb{Z}^{d}$ with $x_{k}^{\prime}=y_{k}^{\prime}=0$.

\subsubsection{Limit Mean Energy Current}

Now let $u(x, t)$ be the random solution to (1.2) with the initial measure $\mu_{0}$ satisfying S0-S3. Then the bounds E1 and (6.3) (see below) imply for the mathematical expectation:

$$
\begin{aligned}
E j^{k}\left(x^{\prime}, t\right)= & \frac{1}{2} \sum_{y^{\prime}}\left(\sum_{m \leqslant-1, p \geqslant 0} \operatorname{tr}\left[Q_{t}^{10}\left(x+m e_{k}, y^{\prime}+p e_{k}\right) V^{T}\left(x^{\prime}-y^{\prime}+(m-p) e_{k}\right)\right]\right. \\
& \left.-\sum_{m \geqslant 0, p \leqslant-1} \operatorname{tr}\left[Q_{t}^{10}\left(x^{\prime}+m e_{k}, y^{\prime}+p e_{k}\right) V^{T}\left(x^{\prime}-y^{\prime}+(m-p) e_{k}\right)\right]\right)
\end{aligned}
$$


Therefore, from the convergence (1.11) it follows that in the limit $t \rightarrow \infty$ we get

$$
\begin{aligned}
& E j^{k}\left(x^{\prime}, t\right) \rightarrow j_{\infty}^{k} \\
& =\frac{1}{2} \sum_{y^{\prime}}\left(\sum_{m \leqslant-1, p \geqslant 0} \operatorname{tr}\left[q_{\infty}^{10}\left(x^{\prime}-y^{\prime}+(m-p) e_{k}\right) V^{T}\left(x^{\prime}-y^{\prime}+(m-p) e_{k}\right)\right]\right. \\
& \left.\quad-\sum_{m \geqslant 0, p \leqslant-1} \operatorname{tr}\left[q_{\infty}^{10}\left(x^{\prime}-y^{\prime}+(m-p) e_{k}\right) V^{T}\left(x^{\prime}-y^{\prime}+(m-p) e_{k}\right)\right]\right) .
\end{aligned}
$$

Denote by $x^{\prime}-y^{\prime}=: z^{\prime}, m-p=: s$ and changing the order of the summation in the series we get (taking into account that $\hat{V}^{*}(\theta)=\hat{V}(\theta)$ )

$$
\begin{aligned}
j_{\infty}^{k} & =-\frac{1}{2} \sum_{z^{\prime}} \sum_{s \in \mathbb{Z}^{1}} \operatorname{tr}\left[q_{\infty}^{10}\left(z^{\prime}+s e_{k}\right) V^{T}\left(z^{\prime}+s e_{k}\right)\right] s \\
& =-\frac{1}{2} \sum_{z \in \mathbb{Z}^{d}} \operatorname{tr}\left[q_{\infty}^{10}(z) z_{k} V^{T}(z)\right] \\
& =-i \frac{(2 \pi)^{-d}}{2} \operatorname{tr} \int_{T^{d}} \hat{q}_{\infty}^{10}(\theta) \partial_{k} \hat{V}(\theta) d \theta, \quad k=1, \ldots, d .
\end{aligned}
$$

\subsection{Gibbs Measures}

\subsubsection{Definition of the Gibbs Measures}

Formally Gibbs measures $g_{ \pm}$are

$$
g_{ \pm}\left(d u_{0}, d v_{0}\right)=\frac{1}{Z_{ \pm}} e^{-\frac{\beta_{ \pm}}{2} \Sigma_{x}\left(\left|v_{0}(x)\right|^{2}+\left\langle\mathscr{r} u_{0}, u_{0}\right\rangle\right)} \prod_{x} d u_{0}(x) d v_{0}(x),
$$

where $\beta_{ \pm}=T_{ \pm}^{-1}, T_{ \pm} \geqslant 0$ are the corresponding absolute temperatures. We introduce the Gibbs measures $g_{ \pm}$as the Gaussian measures with the correlation matrices defined by their Fourier transform as

$$
\hat{q}_{ \pm}^{00}(\theta)=T_{ \pm} \hat{V}^{-1}(\theta), \quad \hat{q}_{ \pm}^{11}(\theta)=T_{ \pm}\left(\delta_{k l}\right)_{k, l \in \bar{n}}, \quad \hat{q}_{ \pm}^{01}(\theta)=\hat{q}_{ \pm}^{10}(\theta)=0 .
$$

Let $H_{\alpha}\left(\mathbb{Z}^{d}\right)$ be the Banach space of the vector-valued functions $u(x) \in \mathbb{R}^{n}$ with the finite norm

$$
\|u\|_{\alpha}^{2} \equiv \sum_{x \in \mathbb{Z}^{d}}\left(1+|x|^{2}\right)^{\alpha}|u(x)|^{2}<\infty .
$$

Let us fix arbitrary $\alpha<-d / 2$. Introduce the Gaussian Borel probability measures $g_{ \pm}^{0}(d u), g_{ \pm}^{1}(d v)$ in spaces $H_{\alpha}\left(\mathbb{Z}^{d}\right)$ with characteristic functionals $\left(\beta_{ \pm}=1 / T_{ \pm}\right)$ 


$$
\begin{aligned}
& \hat{g}_{ \pm}^{0}(\psi)=\int \exp \{i\langle u, \psi\rangle\} g_{ \pm}^{0}(d u)=\exp \left\{-\frac{\left\langle\mathscr{V}^{-1} \psi, \psi\right\rangle}{2 \beta_{ \pm}}\right\} \\
& \hat{g}_{ \pm}^{1}(\psi)=\int \exp \{i\langle v, \psi\rangle\} g_{ \pm}^{1}(d v)=\exp \left\{-\frac{\langle\psi, \psi\rangle}{2 \beta_{ \pm}}\right\} \\
& \psi \in D \equiv C_{0}\left(\mathbb{Z}^{d}\right) \otimes \mathbb{R}^{n}
\end{aligned}
$$

By the Minlos theorem, ${ }^{(3)}$ the Borel probability measures $g_{ \pm}^{0}, g_{ \pm}^{1}$ exist in the spaces $H_{\alpha}\left(\mathbb{Z}^{d}\right)$ because formally we have

$$
\begin{aligned}
\int\|u\|_{\alpha}^{2} g_{ \pm}^{0}(d u) & =\sum_{x \in \mathbb{Z}^{d}}\left(1+|x|^{2}\right)^{\alpha} \sum_{i=1}^{n} \int u_{i}(x) u_{i}(x) g_{ \pm}^{0}(d u) \\
& =\sum_{x \in \mathbb{Z}^{d}}\left(1+|x|^{2}\right)^{\alpha} \operatorname{tr} q_{ \pm}^{00}(0)<\infty
\end{aligned}
$$

since $\alpha<-d / 2$ and

$$
\operatorname{tr} q_{ \pm}^{00}(0)=(2 \pi)^{-d} \int_{T^{d}} \operatorname{tr} \hat{q}_{ \pm}^{00}(\theta) d \theta=T_{ \pm}(2 \pi)^{-d} \int_{T^{d}} \operatorname{tr} \hat{V}^{-1}(\theta) d \theta<\infty
$$

The last bound is obvious if $\mathscr{C}_{0}=\varnothing$ and it follows from condition E6 if $\mathscr{C}_{0} \neq \varnothing$. Similarly,

$$
\int\|v\|_{\alpha}^{2} g_{ \pm}^{1}(d v)=T_{ \pm} n \sum_{x \in \mathbb{Z}^{d}}\left(1+|x|^{2}\right)^{\alpha}<\infty, \quad \alpha<-d / 2 .
$$

Finally, we define the Gibbs measures $g_{ \pm}(d Y)$ as the Borel probability measures $g_{ \pm}^{0}(d u) \times g_{ \pm}^{1}(d v)$ in $\left\{Y \in \mathscr{H}_{\alpha}: Y=(u, v)\right\}$. Let $g_{0}(d Y)$ be a "twotemperature" Borel probability measure in $\mathscr{H}_{\alpha}$ that is constructed in Section 2.5.2 with $\mu_{ \pm}(d Y)=g_{ \pm}(d Y)$ and $Y_{0}$ be a random function with distribution $g_{0}$. Denote by $g_{t}$ the distribution of $U(t) Y_{0}, t \in \mathbb{R}$. Now we assume, in addition, that $\mathscr{C}_{0}=\varnothing$, i.e., (cf. condition E6)

$$
\operatorname{det} \hat{V}(\theta) \neq 0, \quad \forall \theta \in T^{d} .
$$

Note that in the case of canonical Gibbs measures condition E5' is fulfilled (see Remark 2.9(iii)). Indeed, by (4.3) we have

$$
\begin{aligned}
& p_{k l}^{00}(\theta) \equiv\left(B^{*}(\theta) \hat{q}_{ \pm}^{00}(\theta) B(\theta)\right)_{k l}=T_{ \pm}\left(B^{*}(\theta) \hat{V}^{-1}(\theta) B(\theta)\right)_{k l}=T_{ \pm} \omega_{k}^{-2}(\theta) \delta_{k l}, \\
& p_{k l}^{11}(\theta) \equiv\left(B^{*}(\theta) \hat{q}_{ \pm}^{11}(\theta) B(\theta)\right)_{k l}=T_{ \pm} \delta_{k l} .
\end{aligned}
$$

Hence, $p_{k l}^{i j}(\theta) \equiv\left(B^{*}(\theta) \hat{q}_{ \pm}^{i j}(\theta) B(\theta)\right)_{k l}=0$ for $k \neq l, \forall i, j$. 
Theorem 4.1. Let conditions E1-E4, (4.4) hold and $\alpha<-d / 2$. Then there exists a Gaussian Borel probability measure $g_{\infty}$ on $\mathscr{H}_{\alpha}$ such that

$$
g_{t} \stackrel{\mathscr{H}_{\alpha}}{\longrightarrow} g_{\infty}, \quad t \rightarrow \infty
$$

Proof. Let us denote by $Q_{t}(x, y)$ the covariance matrix of measure $g_{t}$, $t \in \mathbb{R}$. Note that owing to (3.6), the matrix $Q_{0}(x, y)$ is a "linear combination" of $q_{ \pm}(x-y)$. Hence, $Q_{0}(x, y)$ satisfies conditions $\mathrm{S} 0-\mathrm{S} 2$. Therefore, by (4.3) we have

$$
\left|Q_{0}(x, y)\right| \leqslant C_{1}+\sum_{ \pm} C_{ \pm}\left|q_{ \pm}^{00}(x-y)\right|, \quad x, y \in \mathbb{Z}^{d}
$$

Condition (4.4) implies

$$
\left|q_{ \pm}^{00}(z)\right|=T_{ \pm}\left|F_{\theta \rightarrow z}^{-1}\left[\hat{V}^{-1}(\theta)\right]\right| \sim(1+|z|)^{-N}, \quad \forall N \in \mathbb{N} .
$$

Hence, Lemma 6.1 and Proposition 7.1 (with condition E5' instead of E5, see Remark 2.9(iii)) are applicable to the correlation matrix $Q_{t}(x, y)$, since the proof uses only the bounds of covariance (5.1), (5.2). These bounds are now provided by the decay (4.8) instead of mixing condition S3. Hence, $Q_{t}(x, y) \rightarrow Q_{\infty}(x, y)$, as $t \rightarrow \infty$, and the family of measures $\left\{g_{t}, t \in \mathbb{R}\right\}$ is weakly compact in $\mathscr{H}_{\alpha}, \alpha<-d / 2$. Hence, the convergence (4.7) holds because $g_{t}$ are Gaussian measures.

\subsubsection{Limit Covariance and Energy Current for the Gibbs Measures}

Now we rewrite the limit covariance $\hat{q}_{\infty}(\theta)$ and the limit mean energy current $j_{\infty}$ defined by (4.2) in the case of the initial measure $\mu_{0}=g_{0}$ with $\mu_{ \pm}=g_{ \pm}$defined above. At first, by (2.19)-(2.21) and (4.3) we have

$$
M_{0}^{+}(\theta)=\bar{T}\left(\begin{array}{cc}
\hat{V}^{-1}(\theta) & 0 \\
0 & 1
\end{array}\right), \quad M_{0}^{-}(\theta)=\Delta T\left(\begin{array}{cc}
0 & \Omega^{-1}(\theta) \\
-\Omega^{-1}(\theta) & 0
\end{array}\right),
$$

where $\bar{T}:=\frac{T_{+}+T_{-}}{2}, \Delta T:=\frac{T_{+}-T_{-}}{2}$. Therefore, from Lemma 2.2(iv) and (2.22)-(2.24) we get

$$
\begin{aligned}
& \hat{q}_{\infty}^{00}(\theta)=\bar{T} \hat{V}^{-1}(\theta), \quad \hat{q}_{\infty}^{11}(\theta)=\bar{T} \\
& \hat{q}_{\infty}^{10}(\theta)=-\hat{q}_{\infty}^{01}(\theta)=-i \Delta T \sum_{\sigma=1}^{s+1} \operatorname{sgn}\left(\frac{\partial \omega_{r_{\sigma}}}{\partial \theta_{d}}(\theta)\right) \omega_{r_{\sigma}}^{-1}(\theta) \Pi_{\sigma}(\theta) .
\end{aligned}
$$


Substituting $\hat{q}_{\infty}^{10}(\theta)$ from (4.10) in the r.h.s. of (4.2), we obtain by (2.7)

$$
\begin{aligned}
j_{\infty}^{k}= & -\frac{\Delta T}{2(2 \pi)^{d}} \operatorname{tr} \int_{T^{d}}\left(\sum_{\sigma=1}^{s+1} \operatorname{sgn}\left(\frac{\partial \omega_{r_{\sigma}}}{\partial \theta_{d}}(\theta)\right) \omega_{r_{\sigma}}^{-1}(\theta) \Pi_{\sigma}(\theta)\right) \\
& \times \frac{\partial}{\partial \theta_{k}}\left(\sum_{\sigma^{\prime}=1}^{s+1} \omega_{r_{\sigma^{\prime}}}^{2}(\theta) \Pi_{\sigma^{\prime}}(\theta)\right) d \theta .
\end{aligned}
$$

Since $\Pi_{\sigma}(\theta)$ are the orthogonal projections, we have

$$
\operatorname{tr}\left[\Pi_{\sigma}(\theta) \Pi_{\sigma^{\prime}}(\theta)\right]= \begin{cases}0, & \text { if } \sigma \neq \sigma^{\prime}, \\ r_{\sigma}, & \text { if } \sigma=\sigma^{\prime} .\end{cases}
$$

Moreover, $\operatorname{tr}\left[\Pi_{\sigma}(\theta) \partial_{k} \Pi_{\sigma^{\prime}}(\theta)\right]=0, k=1, \ldots, d$. Hence, we get

$$
\begin{aligned}
j_{\infty}^{k} & =-\frac{\Delta T}{(2 \pi)^{d}} \sum_{\sigma=1}^{s+1} \int_{T^{d}} r_{\sigma} \operatorname{sgn}\left(\frac{\partial \omega_{r_{\sigma}}}{\partial \theta_{d}}(\theta)\right) \frac{\partial \omega_{r_{\sigma}}}{\partial \theta_{k}}(\theta) d \theta \\
& =-\frac{\Delta T}{(2 \pi)^{d}} \sum_{\gamma \in \bar{n}} \int_{T^{d}} \operatorname{sgn}\left(\frac{\partial \omega_{\gamma}}{\partial \theta_{d}}(\theta)\right) \frac{\partial \omega_{\gamma}}{\partial \theta_{k}}(\theta) d \theta .
\end{aligned}
$$

Remark 4.2. (i) From (4.12) it follows that $j_{\infty}^{d}=-\frac{\Delta T}{(2 \pi)^{d}} \sum_{\gamma \in \bar{n}}$ $\int_{T^{d}}\left|\frac{\partial \omega_{\gamma}}{\partial \theta_{d}}(\theta)\right| d \theta<0$ if $T_{+}>T_{-}$.

(ii) In some particular cases we have $j_{\infty}^{k}=0$ for $k=1, \ldots, d-1$ : for example, (a) if each $\omega_{\gamma}(\theta)$ is even on every variable $\theta_{1}, \ldots, \theta_{d-1}$, or (b) if $\operatorname{sgn}\left(\frac{\partial \omega_{\gamma}}{\partial \theta_{d}}(\theta)\right)$ depends only on variable $\theta_{d}$. For instance, (a) and (b) hold for the nearest neighbor crystal, what follows by (3.2).

(iii) $\hat{q}_{\infty}^{10}$ generally is a discontinuous function by (4.10). Therefore, $q_{\infty}^{10}(x)$ decays as a negative power of $|x|$. The exponential decay is impossible in contrast with ref. 20.

\section{BOUNDS FOR INITIAL COVARIANCE}

Definition 5.1. By $l^{p} \equiv l^{p}\left(\mathbb{Z}^{d}\right) \otimes \mathbb{R}^{n}, p \geqslant 1, n \geqslant 1$, we denote the space of sequences $f(k)=\left(f_{1}(k), \ldots, f_{n}(k)\right)$ endowed with norm $\|f\|_{l^{p}}=$ $\left(\sum_{k \in \mathbb{Z}^{d}}|f(k)|^{p}\right)^{1 / p}$.

The next proposition reflects the mixing property in the Fourier transforms $\hat{q}_{ \pm}^{i j}$ of initial correlation functions $q_{ \pm}^{i j}$. Condition S2 implies that $q_{ \pm}^{i j}(z)$ are bounded functions. Therefore, its Fourier transform generally belongs to the Schwartz space of tempered distributions. 
Proposition 5.2. Let conditions S0-S3 hold. Then

(i) For $i, j=0,1$, the following bounds hold

$$
\begin{array}{lll}
\sum_{y \in \mathbb{Z}^{d}}\left|Q_{0}^{i j}(x, y)\right| \leqslant C<\infty & \text { for all } & x \in \mathbb{Z}^{d}, \\
\sum_{x \in \mathbb{Z}^{d}}\left|Q_{0}^{i j}(x, y)\right| \leqslant C<\infty & \text { for all } & y \in \mathbb{Z}^{d} .
\end{array}
$$

Here the constant $C$ does not depend on $x, y \in \mathbb{Z}^{d}$.

(ii) $\hat{q}_{ \pm}^{i j} \in C\left(T^{d}\right), i, j=0,1$.

Proof ad (i). Conditions S0, S2, and S3 imply by ref. 17, Lemma 17.2.3 (or Lemma 9.4(i) below):

$$
\left|Q_{0}^{i j}(x, y)\right| \leqslant C e_{0} \varphi^{1 / 2}(|x-y|), \quad x, y \in \mathbb{Z}^{d} .
$$

Hence, (2.14) implies (5.1):

$$
\sum_{y \in \mathbb{Z}^{d}}\left|Q_{0}^{i j}(x, y)\right| \leqslant C e_{0} \sum_{z \in \mathbb{Z}^{d}} \varphi^{1 / 2}(|z|)<\infty .
$$

Proof ad (ii). The bound (5.3) and condition (1.8) imply the following bound:

$$
\left|q_{ \pm}^{i j}(z)\right| \leqslant C e_{0} \varphi^{1 / 2}(|z|), \quad z \in \mathbb{Z}^{d} .
$$

Hence, from (2.14) it follows that $q_{ \pm}^{i j}(z) \in l^{1}$, what implies $\hat{q}_{ \pm}^{i j} \in C\left(T^{d}\right)$.

Corollary 5.3. Proposition 5.2(i) implies, by the Shur lemma, that for any $\Phi, \Psi \in l^{2}$ the following bound holds:

$$
\left|\left\langle Q_{0}(x, y), \Phi(x) \otimes \Psi(y)\right\rangle\right| \leqslant C\|\Phi\|_{l^{2}}\|\Psi\|_{l^{2}} .
$$

\section{COMPACTNESS OF MEASURES FAMILY}

Proposition 2.10 follows from the bound (6.1) by the Prokhorov Theorem, ref. 28, Lemma II.3.1 using the method of ref. 28, Theorem XII.5.2, since the embedding $\mathscr{H}_{\alpha} \subset \mathscr{H}_{\beta}$ is compact if $\alpha>\beta$.

Lemma 6.1. Let conditions $\mathrm{S} 0, \mathrm{~S} 2, \mathrm{~S} 3$ hold and $\alpha<-d / 2$. Then the following bounds hold

$$
\sup _{t \geqslant 0} E\left\|U(t) Y_{0}\right\|_{\alpha}^{2}<\infty
$$


Proof. Definition (2.1) implies

$$
E\|Y(\cdot, t)\|_{\alpha}^{2}=\sum_{x \in \mathbb{Z}^{d}}\left(1+|x|^{2}\right)^{\alpha}\left(\operatorname{tr} Q_{t}^{00}(x, x)+\operatorname{tr} Q_{t}^{11}(x, x)\right)<\infty .
$$

Since $\alpha<-d / 2$, it remains to prove that

$$
\sup _{t \in \mathbb{R}} \sup _{x, y \in \mathbb{Z}^{d}}\left\|Q_{t}(x, y)\right\| \leqslant C<\infty .
$$

The representation (A.3) gives

$$
\begin{aligned}
Q_{t}^{i j}(x, y) & =E\left(Y^{i}(x, t) \otimes Y^{j}(y, t)\right) \\
& =\sum_{x^{\prime}, y^{\prime} \in \mathbb{Z}^{d}} \sum_{k, l=0,1} \mathscr{G}_{t}^{i k}\left(x-x^{\prime}\right) Q_{0}^{k l}\left(x^{\prime}, y^{\prime}\right) \mathscr{G}_{t}^{j l}\left(y-y^{\prime}\right) \\
& =\left\langle Q_{0}\left(x^{\prime}, y^{\prime}\right), \Phi_{x}^{i}\left(x^{\prime}, t\right) \otimes \Phi_{y}^{j}\left(y^{\prime}, t\right)\right\rangle,
\end{aligned}
$$

where

$$
\Phi_{x}^{i}\left(x^{\prime}, t\right):=\left(\mathscr{G}_{t}^{i 0}\left(x-x^{\prime}\right), \mathscr{G}_{t}^{i 1}\left(x-x^{\prime}\right)\right), \quad x^{\prime} \in \mathbb{Z}^{d}, \quad i=0,1 .
$$

Note that the Parseval identity, (A.5) and condition E6 imply

$$
\begin{aligned}
\left\|\Phi_{x}^{i}(\cdot, t)\right\|_{l^{2}}^{2} & =(2 \pi)^{-d} \int_{T^{d}}\left|\hat{\Phi}_{x}^{i}(\theta, t)\right|^{2} d \theta \\
& =(2 \pi)^{-d} \int_{T^{d}}\left(\left|\hat{\mathscr{G}}_{t}^{i 0}(\theta)\right|^{2}+\left|\hat{\mathscr{G}}_{t}^{i 1}(\theta)\right|^{2}\right) d \theta \leqslant C_{0}<\infty .
\end{aligned}
$$

Then Corollary 5.3 gives

$$
\begin{aligned}
\left|Q_{t}^{i j}(x, y)\right| & =\left|\left\langle Q_{0}\left(x^{\prime}, y^{\prime}\right), \Phi_{x}^{i}\left(x^{\prime}, t\right) \otimes \Phi_{y}^{j}\left(y^{\prime}, t\right)\right\rangle\right| \\
& \leqslant C\left\|\Phi_{x}^{i}(\cdot, t)\right\|_{l^{2}}\left\|\Phi_{y}^{j}(\cdot, t)\right\|_{l^{2}} \leqslant C_{1}<\infty,
\end{aligned}
$$

where the constant $C_{1}$ does not depend on $x, y \in \mathbb{Z}^{d}, t \in \mathbb{R}$.

\section{7. “CUTTING OUT" OF CRITICAL SPECTRUM}

We reduce the proof of the convergences (1.11) and (1.12) by a suitable spectral analysis.

\subsection{Equicontinuity of Covariance}

Obviously, (1.11) is equivalent to the next proposition. 
Proposition 7.1. Let conditions E1-E6 and S0-S3 hold. Then $\forall \Psi \in \mathscr{D}$

$$
\mathscr{Q}_{t}(\Psi, \Psi) \rightarrow \mathscr{Q}_{\infty}(\Psi, \Psi), \quad t \rightarrow \infty .
$$

Let us show that we can restrict ourselves $\Psi \in \mathscr{D}^{0}$, where $\mathscr{D}^{0}$ is a subset of functions $\Psi \in \mathscr{D}$ with vanishing spectrum in a neighborhood of a critical set $\mathscr{C} \subset T^{d}$. For $k=1, \ldots, n$ define the sets

$$
Z_{k}:=\left\{\theta \in T^{d} \backslash \mathscr{C}_{*}: \nabla_{\theta_{d}} \omega_{k}(\theta)=0\right\} .
$$

Definition 7.2. (i) The critical set $\mathscr{C}:=\mathscr{C}_{0} \cup \mathscr{C}_{*} \cup\left(\bigcup_{1}^{n} Z_{k}\right) \cup\left(\bigcup_{1}^{n} \mathscr{C}_{k}\right)$ (see E4).

(ii) $\mathscr{D}^{0}:=\{\Psi \in \mathscr{D}: \hat{\Psi}(\theta)=0$ in a neighborhood of $\mathscr{C}\}$.

The next lemma plays the central role in our arguments although its proof is similar to the proofs of Lemmas 2.2 and 2.3 since $\mathscr{C} \neq T^{d}$.

Lemma 7.3. Let conditions E1-E4 hold. Then mes $\mathscr{C}=0$.

Next, we introduce a norm $\|\cdot\|_{V}$ in the space $\mathscr{D}$ such that (i) $\mathscr{D}^{0}$ is dense in $\mathscr{D}$ in this norm, while (ii) the quadratic forms $\mathscr{Q}_{t}(\Psi, \Psi), t \in \mathbb{R}$, are equicontinuous in this norm. Then it suffices to prove (7.1) for $\Psi \in \mathscr{D}^{0}$ only.

Definition 7.4. $\mathscr{D}_{V}$ is the space $\mathscr{D}$ endowed with the norm

$$
\|\Psi\|_{V}^{2}:=\int_{T^{d}}\left(1+\left\|V^{-1}(\theta)\right\|\right)|\hat{\Psi}(\theta)|^{2} d \theta, \quad \Psi \in \mathscr{D}
$$

which is finite by condition E6.

The set $\mathscr{D}^{0}$ is dense in $\mathscr{D}_{V}$ by Lemma 7.3 and condition E6.

Lemma 7.5. The quadratic forms $\mathscr{Q}_{t}(\Psi, \Psi), t \in \mathbb{R}$, are equicontinuous in $\mathscr{D}_{V}$.

Proof. It suffices to prove the uniform bounds

$$
\sup _{t \in \mathbb{R}}\left|\mathscr{Q}_{t}(\Psi, \Psi)\right| \leqslant C\|\Psi\|_{V}^{2}, \quad \Psi \in \mathscr{D} .
$$

Definition (2.11) implies that $\mathscr{Q}_{t}(\Psi, \Psi):=E|\langle Y(x, t), \Psi(x)\rangle|^{2}$. Note that

$$
\langle Y(x, t), \Psi(x)\rangle=\left\langle Y_{0}(x), \Phi(x, t)\right\rangle,
$$


where $\Phi(\cdot, t):=F^{-1}\left[\hat{\mathscr{G}}_{t}^{*}(\theta) \hat{\Psi}(\theta)\right] . \quad$ Therefore, $\quad \mathscr{Q}_{t}(\Psi, \Psi)=\mathscr{Q}_{0}(\Phi(\cdot, t)$, $\Phi(\cdot, t))$, so

$$
\sup _{t \in \mathbb{R}}\left|\mathscr{Q}_{t}(\Psi, \Psi)\right| \leqslant C \sup _{t \in \mathbb{R}}\|\Phi(\cdot, t)\|_{l^{2}}^{2}
$$

by Corollary 5.3. Finally, by the Parseval identity and (A.5), we get

$$
\|\Phi(\cdot, t)\|_{l^{2}}^{2}=(2 \pi)^{-d} \int_{T^{d}}\left\|\hat{\mathscr{G}}_{t}^{*}(\theta)\right\|^{2}|\hat{\Psi}(\theta)|^{2} d \theta \leqslant C\|\Psi\|_{V}^{2}
$$

\subsection{Equicontinuity of Characteristic Functionals}

The convergence (1.12) also it suffices to prove for $\Psi \in \mathscr{D}^{0}$ only. This follows from the next lemma.

Lemma 7.6. The characteristic functionals $\hat{\mu}_{t}(\Psi), t \in \mathbb{R}$, are equicontinuous in $\mathscr{D}_{V}$.

Proof. This lemma follows immediately from Lemma 7.5 by the Cauchy-Schwartz inequality:

$$
\begin{aligned}
\left|\hat{\mu}_{t}\left(\Psi_{1}\right)-\hat{\mu}_{t}\left(\Psi_{2}\right)\right| & =\left|\int\left(e^{i\left\langle Y, \Psi_{1}\right\rangle}-e^{i\left\langle Y, \Psi_{2}\right\rangle}\right) \mu_{t}(d Y)\right| \\
& \leqslant \int\left|e^{i\left\langle Y, \Psi_{1}-\Psi_{2}\right\rangle}-1\right| \mu_{t}(d Y) \\
& \leqslant \int\left|\left\langle Y, \Psi_{1}-\Psi_{2}\right\rangle\right| \mu_{t}(d Y) \leqslant \sqrt{\int\left|\left\langle Y, \Psi_{1}-\Psi_{2}\right\rangle\right|^{2} \mu_{t}(d Y)} \\
& =\sqrt{\mathscr{Q}_{t}\left(\Psi_{1}-\Psi_{2}, \Psi_{1}-\Psi_{2}\right)} \leqslant C\left\|\Psi_{1}-\Psi_{2}\right\|_{V} .
\end{aligned}
$$

\section{CONVERGENCE OF COVARIANCE FOR NON-CRITICAL SPECTRUM}

We prove Proposition 7.1 for $\Psi \in \mathscr{D}^{0}$. First we split the initial covariance into the following matrices

$$
\begin{aligned}
& Q^{+}(x, y):=\mathbf{q}^{+}(x-y), \\
& Q^{-}(x, y):=\mathbf{q}^{-}(x-y) \operatorname{sgn} y_{d}, \\
& Q^{r}(x, y):=Q_{0}(x, y)-Q^{+}(x, y)-Q^{-}(x, y)
\end{aligned}
$$


where $\mathbf{q}^{+}=\frac{1}{2}\left(q_{+}+q_{-}\right), \mathbf{q}^{-}=\frac{1}{2}\left(q_{+}-q_{-}\right)$. Since the solution $Y(t)$ to Cauchy problem (1.2) admits the representation (A.3), we have

$$
Q_{t}(x, y)=\sum_{x^{\prime}, y^{\prime} \in \mathbb{Z}^{d}}\left(\mathscr{G}_{t}\left(x-x^{\prime}\right) Q_{0}\left(x^{\prime}, y^{\prime}\right) \mathscr{G}_{t}^{T}\left(y-y^{\prime}\right)\right) .
$$

Next introduce the matrices

$$
Q_{t}^{a}(x, y)=\sum_{x^{\prime}, y^{\prime} \in \mathbb{Z}^{d}}\left(\mathscr{G}_{t}\left(x-x^{\prime}\right) Q^{a}\left(x^{\prime}, y^{\prime}\right) \mathscr{G}_{t}^{T}\left(y-y^{\prime}\right)\right), \quad x, y \in \mathbb{Z}^{d}, \quad t>0
$$

for each $a=\{+,-, r\}$, and split $Q_{t}(x, y)$ into three terms: $Q_{t}(x, y)=$ $Q_{t}^{+}(x, y)+Q_{t}^{-}(x, y)+Q_{t}^{r}(x, y)$. Below in Lemmas 8.1, 8.2, 8.4 we will prove the convergence of type (7.1) to a limit for each term $Q_{t}^{a}(x, y)$.

\subsection{Convergence of $Q_{t}^{+}(x, y)$}

Lemma 8.1. $\lim _{t \rightarrow \infty}\left\langle Q_{t}^{+}(x, y), \Psi(x) \otimes \Psi(y)\right\rangle=\left\langle q_{\infty}^{+}(x-y), \Psi(x)\right.$ $\otimes \Psi(y)\rangle$ for any $\Psi \in \mathscr{D}^{0}$, where the matrix $q_{\infty}^{+}$is defined by (2.23).

Proof. At first, let us apply the Fourier transform to the matrix $Q_{t}^{+}(x, y)$ defined by (8.4). Then we have $\hat{Q}_{t}^{+}\left(\theta, \theta^{\prime}\right):=F_{\substack{x \rightarrow \theta \\ y \rightarrow-\theta^{\prime}}} Q_{t}^{+}(x, y)=$ $\hat{\mathscr{G}}_{t}(\theta) \hat{Q}^{+}\left(\theta, \theta^{\prime}\right) \hat{\mathscr{G}}_{t}^{T}\left(-\theta^{\prime}\right)$, where $\hat{Q}^{+}\left(\theta, \theta^{\prime}\right):=F_{\substack{x \rightarrow \theta \\ y \rightarrow-\theta^{\prime}}} Q^{+}(x, y)$. From (8.1) it follows that $\hat{Q}^{+}\left(\theta, \theta^{\prime}\right)=\delta\left(\theta-\theta^{\prime}\right)(2 \pi)^{d} \hat{\mathbf{q}}^{+}(\theta)$. Hence,

$$
\hat{Q}_{t}^{+}\left(\theta, \theta^{\prime}\right)=(2 \pi)^{d} \delta\left(\theta-\theta^{\prime}\right) \hat{\mathscr{G}}_{t}(\theta) \hat{\mathbf{q}}^{+}(\theta) \hat{\mathscr{G}}_{t}^{*}(\theta) .
$$

Here we use that $\hat{\mathscr{G}}_{t}^{T}(-\theta)=\hat{\mathscr{G}}_{t}^{*}(\theta)$ by condition E2. Therefore,

$$
\begin{aligned}
\left\langle Q_{t}^{+}(x, y), \Psi(x) \otimes \Psi(y)\right\rangle & =(2 \pi)^{-2 d}\left\langle\hat{Q}_{t}^{+}\left(\theta, \theta^{\prime}\right), \hat{\Psi}(\theta) \otimes \overline{\hat{\Psi}}\left(\theta^{\prime}\right)\right\rangle \\
& =(2 \pi)^{-d}\left\langle\hat{\mathscr{G}}_{t}(\theta) \hat{\mathbf{q}}^{+}(\theta) \hat{\mathscr{G}}_{t}^{*}(\theta), \hat{\Psi}(\theta) \otimes \bar{\Psi}(\theta)\right\rangle .
\end{aligned}
$$

Further, we choose certain smooth branches of the functions $B(\theta)$ and $\omega_{k}(\theta)$ to apply the stationary phase arguments which require a smoothness in $\theta$. We choose a finite partition of unity

$$
\sum_{m=1}^{M} g_{m}(\theta)=1, \quad \theta \in \operatorname{supp} \hat{\Psi}
$$

where $g_{m}$ are nonnegative functions from $C_{0}^{\infty}\left(T^{d}\right)$ and vanish in a neighborhood of the set $\mathscr{C}$ defined in Definition 7.2(i). Further, using (8.7) we 
rewrite the r.h.s. of (8.6). Applying formulas (A.6), (A.7) for $\hat{\mathscr{G}}_{t}(\theta), \hat{\mathscr{G}}_{t}^{*}(\theta)$, we obtain (see Appendix A)

$$
\begin{aligned}
& \left\langle Q_{t}^{+}(x, y), \Psi(x) \otimes \Psi(y)\right\rangle \\
& \quad=(2 \pi)^{-d} \sum_{m} \int_{T^{d}} g_{m}(\theta)\left(B(\theta) R_{t}(\theta) B^{*}(\theta), \hat{\Psi}(\theta) \otimes \overline{\hat{\Psi}}(\theta)\right) d \theta,
\end{aligned}
$$

where by $R_{t}(\theta)$ we denote the $2 n \times 2 n$ matrix with the entries (cf. (A.13)):

$$
\begin{aligned}
R_{t}(\theta)_{k l}= & \frac{1}{2} \sum_{ \pm}\left\{\cos \left(\omega_{k}(\theta) \pm \omega_{l}(\theta)\right) t\left[B^{*}(\theta)\left(\hat{\mathbf{q}}^{+}(\theta) \mp \hat{C}(\theta) \hat{\mathbf{q}}^{+}(\theta) \hat{C}^{*}(\theta)\right) B(\theta)\right]_{k l}\right. \\
& \left.+\sin \left(\omega_{k}(\theta) \pm \omega_{l}(\theta)\right) t\left[B^{*}(\theta)\left(\hat{C}(\theta) \hat{\mathbf{q}}^{+}(\theta) \pm \hat{\mathbf{q}}^{+}(\theta) \hat{C}^{*}(\theta)\right) B(\theta)\right]_{k l}\right\}
\end{aligned}
$$

By Lemma 2.2 and the compactness arguments, we choose the eigenvalues $\omega_{k}(\theta)$ and the matrix $B(\theta)$ as real-analytic functions inside the supp $g_{m}$ for every $m$ : we do not mark the functions by the index $m$ to not overburden the notations. Now we analyze the Fourier integrals with $g_{m}$.

At first, note that the identities $\omega_{k}(\theta)+\omega_{l}(\theta) \equiv$ const $_{+}$or $\omega_{k}(\theta)-\omega_{l}(\theta)$ $\equiv$ const _ with the const $t_{ \pm} \neq 0$ are impossible by condition E5. Furthermore, the oscillatory integrals with $\omega_{k}(\theta) \pm \omega_{l}(\theta) \not \equiv$ const vanish as $t \rightarrow \infty$. Hence, only the integrals with $\omega_{k}(\theta)-\omega_{l}(\theta) \equiv 0$ contribute to the limit, since $\omega_{k}(\theta)+\omega_{l}(\theta) \equiv 0$ would imply $\omega_{k}(\theta) \equiv \omega_{l}(\theta) \equiv 0$ which is impossible by E4. We enumerate the eigenvalues $\omega_{k}(\theta)$ as in (2.5). Then if $k, l \in\left(r_{\sigma-1}, r_{\sigma}\right]$, we have $\cos \left(\omega_{k}-\omega_{l}\right) t=1$ for $\sigma=1, \ldots, s+1$. By formula (A.15) with $\hat{q}(\theta):=\hat{\mathbf{q}}^{+}(\theta)$ and (8.8), (8.9), we get

$$
\begin{aligned}
& \left\langle Q_{t}^{+}(x, y), \Psi(x) \otimes \Psi(y)\right\rangle \\
& =(2 \pi)^{-d} \sum_{m} \int_{T^{d}} g_{m}(\theta)\left(B(\theta)\left[\chi_{k l}\left(B^{*}(\theta) M_{0}^{+}(\theta) B(\theta)\right)_{k l}\right]_{k, l \in \bar{n}}\right. \\
& \left.\quad \times B^{*}(\theta)+\cdots, \hat{\Psi}(\theta) \otimes \overline{\hat{\Psi}}(\theta)\right) d \theta \\
& =(2 \pi)^{-d} \int_{T^{d}}\left(\hat{q}_{\infty}^{+}(\theta), \hat{\Psi}(\theta) \otimes \bar{\Psi}(\theta)\right) d \theta+\cdots,
\end{aligned}
$$

where $M_{0}^{+}(\theta)$ is defined in (2.19), “..." stands for the oscillatory integrals which contain $\cos \left(\omega_{k}(\theta) \pm \omega_{l}(\theta)\right) t$ and $\sin \left(\omega_{k}(\theta) \pm \omega_{l}(\theta)\right) t$ with $\omega_{k}(\theta) \pm$ $\omega_{l}(\theta) \not \equiv$ const. The oscillatory integrals converge to zero by the LebesgueRiemann Theorem since all the integrands in "..." are summable, and $\nabla\left(\omega_{k}(\theta) \pm \omega_{l}(\theta)\right)=0$ only on the set of the Lebesgue measure zero. The summability follows from Proposition 5.2(ii) and E6 (if $\mathscr{C}_{0} \neq \varnothing$ ) since the 
matrices $B(\theta)$ are unitary. The zero measure follows similarly to (2.4) since $\omega_{k}(\theta) \pm \omega_{l}(\theta) \not \equiv$ const. Lemma 8.1 is proved.

\subsection{Convergence of $Q_{t}^{-}(x, y)$}

Lemma 8.2. $\lim _{t \rightarrow \infty}\left\langle Q_{t}^{-}(x, y), \Psi(x) \otimes \Psi(y)\right\rangle=\left\langle q_{\infty}^{-}(x-y), \Psi(x)\right.$ $\otimes \Psi(y)\rangle$ for any $\Psi \in \mathscr{D}^{0}$, where the matrix $q_{\infty}^{-}$is defined in (2.24).

\section{Proof.}

Step 1. At first we apply the Fourier transform to $Q_{t}^{-}(x, y)$ defined by (8.4):

$$
\hat{Q}_{t}^{-}\left(\theta, \theta^{\prime}\right):=F_{\substack{x \rightarrow \theta \theta \\ y \rightarrow-\theta^{\prime}}} Q_{t}^{-}(x, y)=\hat{\mathscr{G}}_{t}(\theta) \hat{Q}^{-}\left(\theta, \theta^{\prime}\right) \hat{\mathscr{G}}_{t}^{T}\left(-\theta^{\prime}\right),
$$

where $\hat{Q}^{-}\left(\theta, \theta^{\prime}\right):=F_{\substack{x \rightarrow \theta \\ y \rightarrow-\theta^{\prime}}} Q^{-}(x, y)$. Similarly to (8.6) and (8.8) using the partition of unity (8.7) and also formulas (A.10) and (A.11) we obtain

$$
\begin{aligned}
& \left\langle Q_{t}^{-}(x, y), \Psi(x) \otimes \Psi(y)\right\rangle \\
& \quad=(2 \pi)^{-2 d}\left\langle\hat{Q}_{t}^{-}\left(\theta, \theta^{\prime}\right), \hat{\Psi}(\theta) \otimes \overline{\hat{\Psi}}\left(\theta^{\prime}\right)\right\rangle \\
& \quad=(2 \pi)^{-2 d} \sum_{m, m^{\prime}}\left\langle g_{m}(\theta) g_{m^{\prime}}\left(\theta^{\prime}\right) B(\theta) R_{t}\left(\theta, \theta^{\prime}\right) B^{*}\left(\theta^{\prime}\right), \hat{\Psi}(\theta) \otimes \overline{\hat{\Psi}}\left(\theta^{\prime}\right)\right\rangle,
\end{aligned}
$$

where $R_{t}\left(\theta, \theta^{\prime}\right)$ is defined in (A.11) with $\hat{Q}\left(\theta, \theta^{\prime}\right):=\hat{Q}^{-}\left(\theta, \theta^{\prime}\right)$. Second, we have $F_{y \rightarrow \theta}(\operatorname{sgn} y)=i \operatorname{PV}\left(\frac{1}{\operatorname{tg}(\theta / 2)}\right), \theta \in T^{1}$, where PV stands for the Cauchy principal part and $y \in \mathbb{Z}^{1}$. Hence, by (8.2), we obtain

$$
\hat{Q}^{-}\left(\theta, \theta^{\prime}\right)=\delta\left(\bar{\theta}-\bar{\theta}^{\prime}\right)(2 \pi)^{d-1} i \mathrm{PV}\left(\frac{1}{\operatorname{tg}\left(\theta_{d}-\theta_{d}^{\prime}\right) / 2}\right) \hat{\mathbf{q}}^{-}(\theta) .
$$

Here $\bar{\theta}=\left(\theta_{1}, \ldots, \theta_{d-1}\right), \quad \bar{\theta}^{\prime}=\left(\theta_{1}^{\prime}, \ldots, \theta_{d-1}^{\prime}\right), \quad \theta=\left(\bar{\theta}, \theta_{d}\right), \quad \theta^{\prime}=\left(\bar{\theta}^{\prime}, \theta_{d}^{\prime}\right) \in T^{d}$. Note that the Fourier transform of $Q_{t}^{-}(x, y)$ is more singular than of $Q_{t}^{+}(x, y)$ (cf. formulas (8.5) and (8.11), (8.13)). Therefore it is of key importance that we can restrict ourselves by the functions $\Psi \in \mathscr{D}^{0}$. Further, (8.13) and (A.11) with $\hat{Q}\left(\theta, \theta^{\prime}\right):=\hat{Q}^{-}\left(\theta, \theta^{\prime}\right)$ imply

$$
\begin{aligned}
R_{t}\left(\theta, \theta^{\prime}\right)_{k l}= & \delta\left(\bar{\theta}-\bar{\theta}^{\prime}\right)(2 \pi)^{d-1} i \operatorname{PV}\left(\frac{1}{\operatorname{tg}\left(\theta_{d}-\theta_{d}^{\prime}\right) / 2}\right) \\
& \cdot \sum_{ \pm}\left\{\cos \omega_{k l}^{ \pm} t\left(M_{1}^{ \pm}\left(\theta, \theta^{\prime}\right)\right)_{k l}+\sin \omega_{k l}^{ \pm} t\left(M_{2}^{ \pm}\left(\theta, \theta^{\prime}\right)\right)_{k l}\right\} .
\end{aligned}
$$

Here $\omega_{k l}^{ \pm} \equiv \omega_{k l}^{ \pm}\left(\theta, \theta^{\prime}\right):=\omega_{k}(\theta) \pm \omega_{l}\left(\theta^{\prime}\right), M_{1}^{ \pm}\left(\theta, \theta^{\prime}\right):=B^{*}(\theta) \frac{1}{2}\left(\hat{\mathbf{q}}^{-}(\theta) \mp \hat{C}(\theta)\right.$ $\left.\times \hat{\mathbf{q}}^{-}(\theta) \hat{C}^{*}\left(\theta^{\prime}\right)\right) B\left(\theta^{\prime}\right), M_{2}^{ \pm}\left(\theta, \theta^{\prime}\right):=B^{*}(\theta) \frac{1}{2}\left(\hat{C}(\theta) \hat{\mathbf{q}}^{-}(\theta) \pm \hat{\mathbf{q}}^{-}(\theta) \hat{C}^{*}\left(\theta^{\prime}\right)\right) B\left(\theta^{\prime}\right)$. 
Let us analyze the summands in the r.h.s. of (8.12). Since $\cos \left(\omega_{k l}^{ \pm} t\right)=$ $\frac{e^{i \omega \omega^{ \pm}} k l^{t}+e^{-i \omega^{ \pm} k l^{t}}}{2}$ and $\sin \left(\omega_{k l}^{ \pm} t\right)=\frac{e^{i \omega^{ \pm} k l^{t}-e^{-i \omega^{ \pm}} k l^{t}}}{2 i}$ it suffices to prove the convergence for arising integrals $I_{k l}^{ \pm}(t)$ resp. $J_{k l}^{ \pm}(t)$ with $e^{i \omega_{k l}^{ \pm} t}$ resp. $e^{-i \omega_{k l}^{ \pm} t}$ (see Step 2 resp. Step 3).

Step 2. First, we consider the integrals $I_{k l}^{ \pm}(t)$. Let us denote, for simplicity of exposition, $g_{m} \equiv g_{m}(\theta), g_{m^{\prime}} \equiv g_{m^{\prime}}\left(\theta^{\prime}\right)$, and $\hat{\Psi}_{r}(\theta):=\left(\hat{\Psi}_{r}^{0}(\theta)\right.$, $\left.\hat{\Psi}_{r}^{1}(\theta)\right)$. Also let us denote by $p_{k l}\left(\theta, \theta^{\prime}\right)$ one of the expressions $B_{r k}(\theta) \times$ $\left(M_{i}^{ \pm}\left(\theta, \theta^{\prime}\right)\right)_{k l} B_{k s}^{*}\left(\theta^{\prime}\right)$ with either + or - , and some $i=1,2, r, s \in \bar{n}$. Then (8.12) and (8.14) give,

$$
\begin{aligned}
I_{k l}^{ \pm}(t):= & (2 \pi)^{-2 d}\left\langle g_{m} g_{m^{\prime}} \delta\left(\bar{\theta}-\bar{\theta}^{\prime}\right)(2 \pi)^{d-1} i \mathrm{PV} \frac{1}{\operatorname{tg}\left(\theta_{d}-\theta_{d}^{\prime}\right) / 2}\right. \\
& \left.\times e^{i \omega_{k l}^{ \pm} t} p_{k l}\left(\theta, \theta^{\prime}\right), \hat{\Psi}_{r}(\theta) \otimes \overline{\hat{\Psi}}_{s}\left(\theta^{\prime}\right)\right\rangle \\
= & (2 \pi)^{-d-1} i \int_{T^{d}} g_{m} e^{i \omega_{k}(\theta) t} \overline{\hat{\Psi}}_{r}(\theta) \\
& \times\left(\left.\mathrm{PV} \int_{T^{1}} g_{m^{\prime}} e^{ \pm i \omega_{l}\left(\theta^{\prime}\right) t} \frac{p_{k l}\left(\theta, \theta^{\prime}\right) \hat{\Psi}_{s}\left(\theta^{\prime}\right)}{\operatorname{tg}\left(\theta_{d}-\theta_{d}^{\prime}\right) / 2}\right|_{\theta^{\prime}=\left(\bar{\theta}, \theta_{d}^{\prime}\right)} d \theta_{d}^{\prime}\right) d \theta .
\end{aligned}
$$

The integral with PV in the r.h.s. of (8.15) exists since $\omega_{l}\left(\theta^{\prime}\right)$ are analytic inside the supp $g_{m^{\prime}}\left(\theta^{\prime}\right)$. Changing variables $\theta_{d}^{\prime} \rightarrow \theta_{d}-\theta_{d}^{\prime}=\xi$ in the inner integral in the r.h.s. of (8.15) we obtain

$$
\begin{aligned}
I_{k l}^{ \pm}(t)= & (2 \pi)^{-d-1} i \int_{T^{d}} g_{m} e^{i \omega_{k}(\theta) t} \hat{\hat{\Psi}}_{r}(\theta) \\
& \times\left(\left.\mathrm{PV} \int_{T^{1}} g_{m^{\prime}} e^{ \pm i \omega_{l}\left(\theta^{\prime}\right) t} \frac{p_{k l}\left(\theta, \theta^{\prime}\right) \hat{\Psi}_{s}\left(\theta^{\prime}\right)}{\operatorname{tg}(\xi / 2)}\right|_{\theta^{\prime}=\left(\bar{\theta}, \theta_{d}-\xi\right)} d \xi\right) d \theta .
\end{aligned}
$$

From Definition 7.2 it follows that $\nabla_{\theta_{d}^{\prime}} \omega_{l}\left(\theta^{\prime}\right) \neq 0$ for $\theta^{\prime} \in \operatorname{supp} g_{m^{\prime}} \subset$ supp $\hat{\Psi}$. Next lemma follows from ref. 1, Proposition A.4(i), (ii). Then

Lemma 8.3. Let $\nabla_{\theta_{d}} \omega_{l}(\theta) \neq 0$ for $\theta \in \operatorname{supp} g_{m^{\prime}}$ and $p(\theta) \in C^{1}\left(T^{d}\right)$.

$$
\begin{aligned}
P_{l}(\theta, t): & =\mathrm{PV} \int_{T^{1}} g_{m^{\prime}}\left(\bar{\theta}, \theta_{d}-\xi\right) \frac{e^{ \pm i \omega_{l}\left(\bar{\theta}, \theta_{d}-\xi\right) t}}{\operatorname{tg}(\xi / 2)} p\left(\bar{\theta}, \theta_{d}-\xi\right) d \xi \\
& =2 \pi i g_{m^{\prime}}(\theta) e^{ \pm i \omega_{l}(\theta) t} p(\theta) \operatorname{sgn}\left(\mp \frac{\partial \omega_{l}}{\partial \theta_{d}}(\theta)\right)+o(1), \quad t \rightarrow+\infty
\end{aligned}
$$


for $\theta \in \operatorname{supp} g_{m^{\prime}}$, and

$$
\sup _{\theta \in T^{d}, t \in \mathbb{R}, l \in \bar{n}}\left|P_{l}(\theta, t)\right|<\infty .
$$

Applying Lemma 8.3 to the inner 1D integral in (8.16), we obtain as $t \rightarrow+\infty$,

$$
\begin{aligned}
I_{k l}^{ \pm}(t)= & -(2 \pi)^{-d} \int_{T^{d}} g_{m}(\theta) g_{m^{\prime}}(\theta) e^{i \omega_{k l}^{ \pm}(\theta, \theta) t} p_{k l}(\theta, \theta) \\
& \times \operatorname{sgn}\left(\mp \frac{\partial \omega_{l}}{\partial \theta_{d}}(\theta)\right) \hat{\Psi}_{r}(\theta) \hat{\Psi}_{s}(\theta) d \theta+o(1),
\end{aligned}
$$

where $\omega_{k l}^{ \pm}(\theta, \theta) \equiv \omega_{k}(\theta) \pm \omega_{l}(\theta)$. Let us discuss the limits of the integrals $I_{k l}^{ \pm}(\theta)$ as $t \rightarrow+\infty$. At first, we note that the identities $\omega_{k l}^{+}(\theta, \theta) \equiv$ const $_{+}$ or $\omega_{k l}^{-}(\theta, \theta) \equiv$ const $_{-}$with the const ${ }_{ \pm} \neq 0$ are impossible by condition E5. On the other hand, the oscillatory integrals with $\omega_{k l}^{ \pm}(\theta, \theta) \not \equiv$ const vanish as $t \rightarrow \infty$ owing to Proposition 5.2(ii), E4, E5, E6 (if $\mathscr{C}_{0} \neq \varnothing$ ) and the Lebesgue-Riemann theorem (as in Lemma 8.1). Hence,

$$
I_{k l}^{+}(t) \rightarrow 0, \quad t \rightarrow+\infty .
$$

Similarly, in the case $\omega_{k l}^{-}(\theta, \theta) \not \equiv 0$, we have $I_{k l}^{-}(t) \rightarrow 0$, as $t \rightarrow \infty$. Therefore, only integrals with $\omega_{k l}^{-}(\theta, \theta) \equiv 0$, i.e., $k, l \in\left(r_{\sigma-1}, r_{\sigma}\right], \sigma=1, \ldots, s+1$ (see (2.5)), contribute to a limit. Finally, by (2.18), we get

$$
\begin{aligned}
I_{k l}^{-}(t)= & -(2 \pi)^{-d} \int_{T^{d}} g_{m} g_{m^{\prime}} \chi_{k l} p_{k l}(\theta, \theta) \\
& \times \operatorname{sgn}\left(\frac{\partial \omega_{k}}{\partial \theta_{d}}(\theta)\right) \overline{\hat{\Psi}}_{r}(\theta) \hat{\Psi}_{s}(\theta) d \theta+o(1), \quad t \rightarrow+\infty .
\end{aligned}
$$

Step 3. Now consider the integrals $J_{k l}^{ \pm}(t)$ of type (8.15) with $e^{-i \omega_{k l}^{ \pm} t}$ instead of $e^{i \omega_{k l}^{ \pm} t}$. Similarly to (8.15)-(8.20), we get

$$
\begin{aligned}
J_{k l}^{+}(t):= & (2 \pi)^{-2 d}\left\langle g_{m} g_{m^{\prime}} \delta\left(\bar{\theta}-\bar{\theta}^{\prime}\right)(2 \pi)^{d-1} i \mathrm{PV} \frac{1}{\operatorname{tg}\left(\theta_{d}-\theta_{d}^{\prime}\right) / 2}\right. \\
& \left.\times e^{-i \omega_{k l}^{+} t} p_{k l}\left(\theta, \theta^{\prime}\right), \hat{\Psi}_{r}(\theta) \otimes \overline{\hat{\Psi}}_{s}\left(\theta^{\prime}\right)\right\rangle \\
= & o(1), \quad t \rightarrow \infty .
\end{aligned}
$$

The same decay as $t \rightarrow+\infty$ is valid if we substitute $\omega_{k l}^{-}$in (8.22) instead of $\omega_{k l}^{+}$for all $k, l \in \bar{n}$ except when $k, l \in\left(r_{\sigma-1}, r_{\sigma}\right]$. For $k, l \in\left(r_{\sigma-1}, r_{\sigma}\right]$ we have 
$\omega_{k}(\theta) \equiv \omega_{l}(\theta)$. Hence, by the arguments of type (8.15)-(8.19) and (8.21), we obtain

$$
\begin{aligned}
J_{k l}^{-}(t)= & (2 \pi)^{-2 d}\left\langle g_{m} g_{m^{\prime}} \delta\left(\bar{\theta}-\bar{\theta}^{\prime}\right)(2 \pi)^{d-1} i \mathrm{PV} \frac{1}{\operatorname{tg}\left(\theta_{d}-\theta_{d}^{\prime}\right) / 2}\right. \\
& \left.\times e^{-i \omega_{k l}^{-} t} p_{k l}\left(\theta, \theta^{\prime}\right), \hat{\Psi}_{r}(\theta) \otimes \hat{\Psi}_{s}\left(\theta^{\prime}\right)\right\rangle \\
= & (2 \pi)^{-d} \int_{T^{d}} g_{m} g_{m^{\prime}} \chi_{k l} p_{k l}(\theta, \theta) \\
& \times \operatorname{sgn}\left(\frac{\partial \omega_{k}}{\partial \theta_{d}}(\theta)\right) \hat{\Psi}_{r}(\theta) \hat{\Psi}_{s}(\theta) d \theta+o(1), \quad t \rightarrow+\infty .
\end{aligned}
$$

From (8.20), (8.22) it follows that for any $k, l \in \bar{n}$ as $t \rightarrow \infty$,

$$
\begin{aligned}
& \left\langle g_{m} g_{m^{\prime}} \delta\left(\bar{\theta}-\bar{\theta}^{\prime}\right)(2 \pi)^{d-1} i \mathrm{PV} \frac{1}{\operatorname{tg}\left(\theta_{d}-\theta_{d}^{\prime}\right) / 2}\right. \\
& \left.\quad \times \cos \left(\omega_{k l}^{ \pm} t\right) p_{k l}\left(\theta, \theta^{\prime}\right), \hat{\Psi}_{r}(\theta) \otimes \bar{\Psi}_{s}\left(\theta^{\prime}\right)\right\rangle=o(1),
\end{aligned}
$$

since the signs in (8.21) and (8.23) are opposite. Similarly, by (8.20) and (8.22), we have

$$
\begin{aligned}
& \left\langle g_{m} g_{m^{\prime}} \delta\left(\bar{\theta}-\bar{\theta}^{\prime}\right)(2 \pi)^{d-1} i \mathrm{PV} \frac{1}{\operatorname{tg}\left(\theta_{d}-\theta_{d}^{\prime}\right) / 2}\right. \\
& \left.\quad \times \sin \left(\omega_{k l}^{+} t\right) p_{k l}\left(\theta, \theta^{\prime}\right), \hat{\Psi}_{r}(\theta) \otimes \overline{\hat{\Psi}}_{s}\left(\theta^{\prime}\right)\right\rangle=o(1), \quad t \rightarrow \infty .
\end{aligned}
$$

The same relation holds if we substitute $\omega_{k l}^{-}$in the 1.h.s. of (8.25) instead of $\omega_{k l}^{+}$for all $k, l \in \bar{n}$ except when $k, l \in\left(r_{\sigma-1}, r_{\sigma}\right]$. At last, using (8.23), we get:

$$
\begin{aligned}
(2 \pi)^{-2 d} & \left\langle g_{m} g_{m^{\prime}} \delta\left(\bar{\theta}-\bar{\theta}^{\prime}\right)(2 \pi)^{d-1} i \mathrm{PV} \frac{1}{\operatorname{tg}\left(\theta_{d}-\theta_{d}^{\prime}\right) / 2}\right. \\
& \left.\times \sin \left(\omega_{k l}^{-} t\right) p_{k l}\left(\theta, \theta^{\prime}\right), \hat{\Psi}_{r}(\theta) \otimes \overline{\hat{\Psi}}_{s}\left(\theta^{\prime}\right)\right\rangle \\
= & (2 \pi)^{-d}\left\langle g_{m} g_{m^{\prime}} \chi_{k l} i \operatorname{sgn}\left(\frac{\partial \omega_{k}}{\partial \theta_{d}}(\theta)\right) p_{k l}(\theta, \theta), \hat{\Psi}_{r}(\theta) \otimes \overline{\hat{\Psi}}_{s}(\theta)\right\rangle \\
& +o(1), \quad t \rightarrow+\infty .
\end{aligned}
$$


Here (see Steps 1 and 2) by $p_{k l}(\theta, \theta)$ we denote $p_{k l}(\theta, \theta) \equiv B_{r k}(\theta)\left(M_{2}^{-}(\theta, \theta)\right)_{k l} B_{k s}^{*}(\theta) \equiv B_{r k}(\theta)\left(B^{*}(\theta) M_{0}^{-}(\theta) B(\theta)\right)_{k l} B_{k s}^{*}(\theta)$, where $M_{0}^{-}(\theta)$ is defined by $(2.20)$.

Step 4. Now we return to the r.h.s. of (8.12). Let us substitute (8.14) in (8.12). Then by (8.24) the summands in the r.h.s. of (8.12) with $\cos \omega_{k l}^{ \pm} t$ tend to zero. Further, by (8.25), (8.26) only integrals with $\sin \omega_{k l}^{-} t, k, l \in$ $\left(r_{\sigma-1}, r_{\sigma}\right], \sigma=1, \ldots, s+1$ contribute to a limit. Finally, (8.12), (8.14), and (8.22)-(8.26) imply,

$\left\langle Q_{t}^{-}(x, y), \Psi(x) \otimes \Psi(y)\right\rangle$

$$
\begin{gathered}
=(2 \pi)^{-d} \sum_{m, m^{\prime}}\left\langle g_{m} g_{m^{\prime}} B(\theta)\left[\chi_{k l} i \operatorname{sgn}\left(\frac{\partial \omega_{k}}{\partial \theta_{d}}(\theta)\right) M_{2}^{-}(\theta, \theta)_{k l}\right]_{k, l \in \bar{n}} B^{*}(\theta),\right. \\
\hat{\Psi}(\theta) \otimes \overline{\hat{\Psi}}(\theta)\rangle+o(1) \\
=(2 \pi)^{-d} \sum_{m, m^{\prime}}\left\langle g_{m} g_{m^{\prime}} \hat{q}_{\infty}^{-}(\theta), \hat{\Psi}(\theta) \otimes \overline{\hat{\Psi}}(\theta)\right\rangle+o(1) \\
=\left\langle q_{\infty}^{-}(x-y), \Psi(x) \otimes \Psi(y)\right\rangle+o(1), \quad t \rightarrow+\infty .
\end{gathered}
$$

\subsection{Convergence of $Q_{t}^{r}(x, y)$}

Lemma 8.4. $\left.\lim _{t \rightarrow \infty}\left\langle Q_{t}^{r}(x, y), \Psi(x) \otimes \Psi(y)\right)\right\rangle=0$ for any $\Psi \in \mathscr{D}^{0}$.

Proof.

Step 1. We develop the method, ref. 1, p. 140. Let us define (as in (7.4))

$$
\Phi\left(x^{\prime}, t\right):=\sum_{x \in \mathbb{Z}^{d}} \mathscr{G}_{t}^{T}\left(x-x^{\prime}\right) \Psi(x)
$$

Then using (8.4) we have,

$$
\begin{aligned}
\left\langle Q_{t}^{r}(x, y), \Psi(x) \otimes \Psi(y)\right\rangle & =\sum_{x^{\prime} \in \mathbb{Z}^{d}} \sum_{y^{\prime} \in \mathbb{Z}^{d}} Q^{r}\left(x^{\prime}, y^{\prime}\right) \Phi\left(x^{\prime}, t\right) \Phi\left(y^{\prime}, t\right) \\
& =\sum_{z^{\prime} \in \mathbb{Z}^{d}} \mathscr{F}_{t}\left(z^{\prime}\right),
\end{aligned}
$$


where

$$
\mathscr{F}_{t}\left(z^{\prime}\right):=\sum_{y^{\prime} \in \mathbb{Z}^{d}} Q^{r}\left(y^{\prime}+z^{\prime}, y^{\prime}\right) \Phi\left(y^{\prime}+z^{\prime}, t\right) \Phi\left(y^{\prime}, t\right)
$$

The estimates (5.3), (5.5), and definition (8.3) imply the same estimate for $Q^{r}(x, y):\left|Q^{r}(x, y)\right| \leqslant C e_{0} \varphi^{1 / 2}(|x-y|)$. Hence, the Cauchy-Schwartz inequality and (7.6) imply

$$
\begin{aligned}
\left|\mathscr{F}_{t}\left(z^{\prime}\right)\right| & \leqslant \sum_{y^{\prime} \in \mathbb{Z}^{d}}\left\|Q^{r}\left(y^{\prime}+z^{\prime}, y^{\prime}\right)\right\|\left|\Phi\left(y^{\prime}+z^{\prime}, t\right)\right|\left|\Phi\left(y^{\prime}, t\right)\right| \\
& \leqslant C \varphi^{1 / 2}\left(\left|z^{\prime}\right|\right) \sum_{y^{\prime} \in \mathbb{Z}^{d}}\left|\Phi\left(y^{\prime}+z^{\prime}, t\right)\right|\left|\Phi\left(y^{\prime}, t\right)\right| \\
& \leqslant C_{1} \varphi^{1 / 2}\left(\left|z^{\prime}\right|\right)\|\Psi\|_{V}^{2},
\end{aligned}
$$

where $\|\Psi\|_{V}^{2}$ is defined by (7.2). Hence, (2.14) and condition E6 imply

$$
\sum_{z^{\prime} \in \mathbb{Z}^{d}}\left|\mathscr{F}_{t}\left(z^{\prime}\right)\right| \leqslant C(\Psi) \sum_{z^{\prime} \in \mathbb{Z}^{d}} \varphi^{1 / 2}\left(\left|z^{\prime}\right|\right) \leqslant C_{1}<\infty,
$$

and the series (8.27) converges uniformly in $t$. Therefore, it suffices to prove that

$$
\lim _{t \rightarrow \infty} \mathscr{F}_{t}\left(z^{\prime}\right)=0 \quad \text { for each } \quad z^{\prime} \in \mathbb{Z}^{d} .
$$

Step 2. Let us prove (8.31). Condition S1 and (8.3) imply that $Q^{r}\left(y^{\prime}+z^{\prime}, y^{\prime}\right)=q^{r}\left(\bar{z}^{\prime}, y_{d}^{\prime}+z_{d}^{\prime}, y_{d}^{\prime}\right)$, where

$$
\lim _{y_{d}^{\prime} \rightarrow \pm \infty} q^{r}\left(\bar{z}^{\prime}, y_{d}^{\prime}+z_{d}^{\prime}, y_{d}^{\prime}\right)=0, \quad \text { for } \quad\left(\bar{z}^{\prime}, z_{d}^{\prime}\right) \in \mathbb{Z}^{d} .
$$

Hence, $\forall \varepsilon>0$ there exists $N \in \mathbb{N}$ so large that $\left|q^{r}\left(\bar{z}^{\prime}, y_{d}^{\prime}+z_{d}^{\prime}, y_{d}^{\prime}\right)\right|<\varepsilon$ for $\left|y_{d}^{\prime}\right|>N$. Respectively, decompose the series (8.28) into two series: $\mathscr{F}_{t}\left(z^{\prime}\right)=$ $\sum_{\bar{y}^{\prime} \in \mathbb{Z}^{d-1}} \sum_{\left|y_{d}^{\prime}\right|>N} \cdots+\sum_{\bar{y}^{\prime} \in \mathbb{Z}^{d-1}} \sum_{\left|y_{d}^{\prime}\right|<N} \cdots$. By (7.6) and condition E6, the first series is estimated by

$$
\begin{aligned}
& \left|\sum_{\bar{y}^{\prime} \in \mathbb{Z}^{d-1}} \sum_{\left|y_{d}^{\prime}\right|>N} q^{r}\left(\bar{z}^{\prime}, y_{d}^{\prime}+z_{d}^{\prime}, y_{d}^{\prime}\right) \Phi\left(y^{\prime}+z^{\prime}, t\right) \Phi\left(y^{\prime}, t\right)\right| \\
& \leqslant \varepsilon \sum_{y^{\prime} \in \mathbb{Z}^{d}}\left|\Phi\left(y^{\prime}, t\right)\right|^{2} \leqslant \varepsilon C(\Psi) .
\end{aligned}
$$

Note that $q^{r}\left(\bar{z}^{\prime}, y_{d}^{\prime}+z_{d}^{\prime}, y_{d}^{\prime}\right)$ does not depend on $\bar{y}^{\prime}$. Then we can rewrite the second series by the Parseval identity as 


$$
\begin{aligned}
\sum_{\left|y_{d}^{\prime}\right|<N} & q^{r}\left(\bar{z}^{\prime}, y_{d}^{\prime}+z_{d}^{\prime}, y_{d}^{\prime}\right) \sum_{\bar{y}^{\prime} \in \mathbb{Z}^{d-1}} \Phi\left(y^{\prime}+z^{\prime}, t\right) \Phi\left(y^{\prime}, t\right) \\
= & (2 \pi)^{-2 d+2} \sum_{\left|y_{d}^{\prime}\right|<N} q^{r}\left(\bar{z}^{\prime}, y_{d}^{\prime}+z_{d}^{\prime}, y_{d}^{\prime}\right) \\
& \times \int_{T^{d-1}} F_{\bar{y}^{\prime} \rightarrow \bar{\theta}}\left[\Phi\left(y^{\prime}+z^{\prime}, t\right)\right] \overline{F_{\bar{y}^{\prime} \rightarrow \bar{\theta}}\left[\Phi\left(y^{\prime}, t\right)\right]} d \bar{\theta} .
\end{aligned}
$$

It remains to prove that the integral in the r.h.s. of (8.34) tends to zero as $t \rightarrow \infty$ for fixed $z^{\prime} \in \mathbb{Z}^{d}$ and $\left|y_{d}^{\prime}\right|<N$. First, let us note that for the integrand in (8.34) the following uniform bound holds,

$$
\begin{aligned}
& \left|F_{\bar{y}^{\prime} \rightarrow \bar{\theta}}\left[\Phi\left(y^{\prime}+z^{\prime}, t\right)\right] \overline{F_{\bar{y}^{\prime} \rightarrow \bar{\theta}}\left[\Phi\left(y^{\prime}, t\right)\right]}\right| \\
& \quad \leqslant G(\bar{\theta}), \quad t \geqslant 0, \quad \text { where } \quad G(\bar{\theta}) \in L^{1}\left(T^{d-1}\right) .
\end{aligned}
$$

Indeed, rewrite the function $F_{\bar{y}^{\prime} \rightarrow \bar{\theta}}\left[\Phi\left(y^{\prime}, t\right)\right]$ in the form

$$
\begin{aligned}
F_{\bar{y}^{\prime} \rightarrow \bar{\theta}}\left[\Phi\left(y^{\prime}, t\right)\right] & =(2 \pi)^{-1} \int_{T^{1}} e^{-i \theta_{d} y_{d}^{\prime}} \hat{\Phi}(\theta, t) d \theta_{d} \\
& =(2 \pi)^{-1} \int_{T^{1}} e^{-i \theta_{d} y_{d}^{\prime}} \hat{\mathscr{G}}_{t}^{*}(\theta) \hat{\Psi}(\theta) d \theta_{d} .
\end{aligned}
$$

Therefore,

$$
\begin{aligned}
& \left|F_{\bar{y}^{\prime} \rightarrow \bar{\theta}}\left[\Phi\left(y^{\prime}+z^{\prime}, t\right)\right] \overline{F_{\bar{y}^{\prime} \rightarrow \bar{\theta}}\left[\Phi\left(y^{\prime}, t\right)\right]}\right| \\
& \quad \leqslant C\left(\int_{T^{1}}\left\|\hat{\mathscr{G}}_{t}^{*}(\theta)\right\||\hat{\Psi}(\theta)| d \theta_{d}\right)^{2} \leqslant C_{1} \int_{T^{1}}\left\|\hat{\mathscr{G}}_{t}^{*}(\theta)\right\|^{2}|\hat{\Psi}(\theta)|^{2} d \theta_{d} \\
& \quad \leqslant C_{2} \int_{T^{1}}\left\|\left(1+\left\|\hat{V}^{-1}(\theta)\right\|\right)\right\||\hat{\Psi}(\theta)|^{2} d \theta_{d}:=G(\bar{\theta})
\end{aligned}
$$

and (8.35) follows from condition E6. Therefore, it suffices to prove that the integrand in the r.h.s. of (8.34) tends to zero as $t \rightarrow \infty$ for a.a. fixed $\bar{\theta} \in T^{d-1}$. We use the finite partition of unity (8.7) (remember that $\Psi \in \mathscr{D}^{0}$ ) and split the function $F_{\bar{y}^{\prime} \rightarrow \bar{\theta}}\left[\Phi\left(y^{\prime}, t\right)\right]$ into the sum of the integrals:

$$
F_{\bar{y}^{\prime} \rightarrow \bar{\theta}}\left[\Phi\left(y^{\prime}, t\right)\right]=\sum_{m} \sum_{ \pm, k \in \bar{n}} \int_{T^{1}} g_{m}(\theta) e^{-i \theta_{d} y_{d}^{\prime}} e^{ \pm i \omega_{k}(\theta) t} a_{k}^{ \pm}(\theta) \hat{\Psi}(\theta) d \theta_{d}, \Psi \in \mathscr{D}^{0}
$$

The eigenvalues $\omega_{k}(\theta)$ and the matrices $a_{k}^{ \pm}(\theta)$ are real-analytic functions inside the $\operatorname{supp} g_{m}$ for every $m$. From Definition 7.2(i) and conditions E4, 
E6 it follows that mes $\left\{\theta_{d} \in T^{1}: \nabla_{\theta_{d}} \omega_{k}(\theta)=0\right\}=0$ for a.a. fixed $\bar{\theta} \in T^{d-1}$. Hence, the integrals in (8.38) vanish as $t \rightarrow \infty$ by the Lebesgue-Riemann theorem.

Finally, Lemmas $8.1,8.2$, and 8.4 imply the convergence (7.1) for $\Psi \in \mathscr{D}^{0}$. Then (7.1) follows for any $\Psi \in \mathscr{D}$ by Lemma 7.5 (see Section 7.1). Proposition 7.1 is proved.

\section{BERNSTEIN'S ARGUMENT}

\subsection{Oscillatory Integrals and Stationary Phase Method}

To prove (1.12) we evaluate $\langle Y(\cdot, t), \Psi\rangle$ by (7.4), where

$$
\Phi(x, t):=F_{\theta \rightarrow x}^{-1}\left[\hat{\mathscr{G}}_{t}^{*}(\theta) \hat{\Psi}(\theta)\right]=(2 \pi)^{-d} \int_{T^{d}} e^{-i \theta x} \hat{\mathscr{G}}_{t}^{*}(\theta) \hat{\Psi}(\theta) d \theta, \quad x \in \mathbb{Z}^{d}
$$

Similarly to (8.38) or (8.8) using the partition of unity (8.7) we get

$$
\Phi(x, t)=\sum_{m} \sum_{ \pm, k \in \bar{n}} \int_{T^{d}} g_{m}(\theta) e^{-i\left(\theta x \pm \omega_{k} t\right)} a_{k}^{ \pm}(\theta) \hat{\Psi}(\theta) d \theta, \quad \Psi \in \mathscr{D}^{0},
$$

where $\omega_{k}(\theta)$ and $a_{k}^{ \pm}(\theta)$ are real-analytic functions inside the supp $g_{m}$ for every $m$.

Note that $\Phi(t):=\Phi(\cdot, t)$ is the solution to the "conjugate" equation (cf (1.3), (1.4))

$$
\dot{\Phi}(t)=\mathscr{A}^{\prime} \Phi(t), \quad t \in \mathbb{R} ; \quad \mathscr{A}^{\prime}=\left(\begin{array}{cc}
0 & -\mathscr{V} \\
1 & 0
\end{array}\right),
$$

which is obvious in the Fourier transform. Therefore, the solutions $Y(t)=$ $\left(Y^{0}(t), Y^{1}(t)\right)$ and $\Phi(t)=\left(\Phi^{0}(t), \Phi^{1}(t)\right)$ to the equations (1.3) and (9.3) coincide up to order of the components. Hence, $\Phi(x, t)$ has corresponding dispersive properties.

We will deduce (1.12) by analyzing the propagation of the solution $\Phi(x, t)$ to Eq. (9.3), in different directions $x=v t$ with $v \in \mathbb{R}^{d}$. For this purpose, we apply the stationary phase method to the oscillatory integral (9.2) along the rays $x=v t, t>0$. Then the phase becomes $\left(\theta v \pm \omega_{k}(\theta)\right) t$, and its stationary points are the solutions to the equations $v=\mp \nabla \omega_{k}(\theta)$.

Recall that we can restrict ourselves by $\Psi \in \mathscr{D}^{0}$, hence $\hat{\Psi}(\theta)=0$ in the points $\theta \in T^{d}$ with degenerate Hessian $D_{k}(\theta)$ (see E4). Therefore, the stationary phase method leads to the following two different types of the asymptotic behavior of $\Phi(v t, t)$ as $t \rightarrow \infty$ : 

Then

(I) For the velocity $v$ inside the light cone: $v= \pm \nabla \omega_{k}(\theta), \theta \in T^{d} \backslash \mathscr{C}$.

$$
\Phi(v t, t)=\mathcal{O}\left(t^{-d / 2}\right) .
$$

(II) For the velocity $v$ outside the light cone: $v \neq \pm \nabla \omega_{k}(\theta)$, $\theta \in T^{d} \backslash \mathscr{C}, k \in \bar{n}$. Then

$$
\Phi(v t, t)=\mathcal{O}\left(t^{-p}\right), \quad \forall p>0 .
$$

The asymptotics of the types (I) and (II) allow us to incorporate the Bernstein-type approach developed in ref. 1 for case $d=1$ and in refs. 7 and 8 for continuous Klein-Gordon and wave equations for $d \geqslant 1$. We formalize (9.4), (9.5) as follows.

Lemma 9.1. For any fixed $\Psi \in \mathscr{D}^{0}$ the following bounds hold:

(i)

$$
\sup _{x \in \mathbb{Z}^{d}}|\Phi(x, t)| \leqslant C t^{-d / 2}
$$

(ii) For any $p>0$ there exist $C_{p}, \gamma>0$ s.t.

$$
|\Phi(x, t)| \leqslant C_{p}(1+|x|+|t|)^{-p}, \quad|x| \geqslant \gamma t .
$$

Proof. Consider $\Phi(x, t)$ along each ray $x=v t$ with arbitrary $v \in \mathbb{R}^{d}$. Substituting to (9.2), we get

$$
\Phi(v t, t)=\sum_{m} \sum_{ \pm, k \in \bar{n}} \int_{T^{d}} g_{m}(\theta) e^{-i\left(\theta v \pm \omega_{k}(\theta)\right) t} a_{k}^{ \pm}(\theta) \hat{\Psi}(\theta) d \theta, \quad \hat{\Psi} \in \mathscr{D}^{0}
$$

This is a sum of oscillatory integrals with the phase functions $\phi_{k}^{ \pm}(\theta)=$ $\theta v \pm \omega_{k}(\theta)$ and the amplitudes $a_{k}^{ \pm}(\theta)$ which are real-analytic functions of the $\theta$ inside the supp $g_{m}$. Since $\omega_{k}(\theta)$ is real-analytic, each function $\phi_{k}^{ \pm}$has no more than a finite number of stationary points $\theta \in \operatorname{supp} g_{m}$, solutions to the equation $v=\mp \nabla \omega_{k}(\theta)$. The stationary points are non-degenerate for $\theta \in \operatorname{supp} g_{m}$ by (8.7), Definition 7.2 and E4 since

$$
\operatorname{det}\left(\frac{\partial^{2} \phi_{k}^{ \pm}}{\partial \theta_{i} \partial \theta_{j}}\right)= \pm D_{k}(\theta) \neq 0, \quad \theta \in \operatorname{supp} g_{m} .
$$

At last, $\hat{\Psi}(\theta)$ is smooth since $\Psi \in \mathscr{D}$. Therefore, $\Phi(v t, t)=\mathcal{O}\left(t^{-d / 2}\right)$ according to the standard stationary phase method. ${ }^{(13,22)}$ This implies the bounds (9.6) in each cone $|x| \leqslant c t$ with any finite $c$. 
Further, denote by $\bar{v}:=\max _{m} \max _{k \in \bar{n}} \max _{\theta \in \operatorname{supp} g_{m}}\left|\nabla \omega_{k}(\theta)\right|$. Then for $|v|>\bar{v}$ the stationary points do not exist on the supp $\hat{\Psi}$. Hence, the integration by parts as in ref. 22 yields $\Phi(v t, t)=\mathcal{O}\left(t^{-p}\right)$ for any $p>0$. On the other hand, the integration by parts in (9.2) implies similar bound $\Phi(x, t)=\mathcal{O}\left((t /|x|)^{l}\right)$ for any $l>0$. Therefore, (9.7) follows with any $\gamma>\bar{v}$. Now the bounds (9.6) follow everywhere.

\section{2. “Rooms-Corridors" Partition}

The remaining constructions in the proof of (1.12) are similar to refs. 7 and 10 . However, the proofs are not identical since here we consider non translation-invariant case.

Let us introduce a "room-corridor" partition of the ball $\left\{x \in \mathbb{Z}^{d}\right.$ : $|x| \leqslant \gamma t\}$, with $\gamma$ from (9.7). For $t>0$ we choose $\Delta_{t}$ and $\rho_{t} \in \mathbb{N}$. Asymptotic relations between $t, \Delta_{t}$, and $\rho_{t}$ are specified below. Let us set $h_{t}=\Delta_{t}+\rho_{t}$ and

$$
a^{j}=j h_{t}, \quad b^{j}=a^{j}+\Delta_{t}, \quad j \in \mathbb{Z}, \quad N_{t}=\left[(\gamma t) / h_{t}\right] .
$$

We call the slabs $R_{t}^{j}=\left\{x \in \mathbb{Z}^{d}:|x| \leqslant N_{t} h_{t}, a^{j} \leqslant x_{d}<b^{j}\right\}$ the "rooms," $C_{t}^{j}=$ $\left\{x \in \mathbb{Z}^{d}:|x| \leqslant N_{t} h_{t}, b^{j} \leqslant x_{d}<a^{j+1}\right\}$ the "corridors" and $L_{t}=\left\{x \in \mathbb{Z}^{d}:|x|\right.$ $\left.>N_{t} h_{t}\right\}$ the "tails." Here $x=\left(x_{1}, \ldots, x_{d}\right), \Delta_{t}$ is the width of a room, and $\rho_{t}$ of a corridor. Let us denote by $\chi_{t}^{j}$ the indicator of the room $R_{t}^{j}$, $\xi_{t}^{j}$ that of the corridor $C_{t}^{j}$, and $\eta_{t}$ that of the tail $L_{t}$. Then

$$
\sum_{t}\left[\chi_{t}^{j}(x)+\xi_{t}^{j}(x)\right]+\eta_{t}(x)=1, \quad x \in \mathbb{Z}^{d},
$$

where the sum $\sum_{t}$ stands for $\sum_{j=-N_{t}}^{N_{t}-1}$. Hence, we get the following Bernstein's type representation:

$$
\left\langle Y_{0}, \Phi(\cdot, t)\right\rangle=\sum_{t}\left[\left\langle Y_{0}, \chi_{t}^{j} \Phi(\cdot, t)\right\rangle+\left\langle Y_{0}, \xi_{t}^{j} \Phi(\cdot, t)\right\rangle\right]+\left\langle Y_{0}, \eta_{t} \Phi(\cdot, t)\right\rangle .
$$

Let us define the random variables $r_{t}^{j}, c_{t}^{j}, l_{t}$ by

$$
r_{t}^{j}=\left\langle Y_{0}, \chi_{t}^{j} \Phi(\cdot, t)\right\rangle, \quad c_{t}^{j}=\left\langle Y_{0}, \xi_{t}^{j} \Phi(\cdot, t)\right\rangle, \quad l_{t}=\left\langle Y_{0}, \eta_{t} \Phi(\cdot, t)\right\rangle .
$$

Then (9.12) becomes

$$
\left\langle Y_{0}, \Phi(\cdot, t)\right\rangle=\sum_{t}\left(r_{t}^{j}+c_{t}^{j}\right)+l_{t} .
$$


Lemma 9.2. Let S0-S3 hold and $\Psi \in \mathscr{D}^{0}$. The following bounds hold for $t>1$ :

$$
\begin{array}{ll}
E\left|r_{t}^{j}\right|^{2} \leqslant C(\Psi) \Delta_{t} / t, & \forall j, \\
E\left|c_{t}^{j}\right|^{2} \leqslant C(\Psi) \rho_{t} / t, & \forall j, \\
E\left|l_{t}\right|^{2} \leqslant C_{p}(\Psi) t^{-p}, & \forall p>0 .
\end{array}
$$

Proof. (9.17) follows from (9.7) and Proposition 5.2(i). We discuss (9.15) only, (9.16) is done in a similar way. Let us express $E\left|r_{t}^{j}\right|^{2}$ in the correlation matrices. Definition (9.13) implies

$$
E\left|r_{t}^{j}\right|^{2}=\left\langle Q_{0}(x, y), \chi_{t}^{j}(x) \Phi(x, t) \otimes \chi_{t}^{j}(y) \Phi(y, t)\right\rangle .
$$

According to (9.6), Eq. (9.18) implies that

$$
\begin{aligned}
E\left|r_{t}^{j}\right|^{2} & \leqslant C t^{-d} \sum_{x, y} \chi_{t}^{j}(x)\left\|Q_{0}(x, y)\right\| \\
& =C t^{-d} \sum_{x} \chi_{t}^{j}(x) \sum_{y \in \mathbb{Z}^{d}}\left\|Q_{0}(x, y)\right\| \leqslant C \Delta_{t} / t,
\end{aligned}
$$

where $\left\|Q_{0}(x, y)\right\|$ stands for the norm of a matrix $\left(Q_{0}^{i j}(x, y)\right)$. Therefore, (9.19) follows by Proposition 5.2(i).

Now we prove the convergence (1.12). As was said, we use a version of the Central Limit Theorem developed by Ibragimov and Linnik. If $\mathscr{Q}_{\infty}(\Psi, \Psi)=0$, the convergence (1.12) is obvious. In fact, then,

$$
\begin{aligned}
\mid E \exp & \left\{i\left\langle Y_{0}, \Phi(\cdot, t)\right\rangle\right\}-\hat{\mu}_{\infty}(\Psi) \mid \\
\quad & =E\left|\exp \left\{i\left\langle Y_{0}, \Phi(\cdot, t)\right\rangle\right\}-1\right| \leqslant E\left|\left\langle Y_{0}, \Phi(\cdot, t)\right\rangle\right| \\
\quad & \leqslant\left(E\left|\left\langle Y_{0}, \Phi(\cdot, t)\right\rangle\right|^{2}\right)^{1 / 2}=\left(\left\langle Q_{0}(x, y), \Phi(x, t) \otimes \Phi(y, t)\right\rangle\right)^{1 / 2} \\
\quad & =\left(\mathscr{Q}_{t}(\Psi, \Psi)\right)^{1 / 2},
\end{aligned}
$$

where $\mathscr{Q}_{t}(\Psi, \Psi) \rightarrow \mathscr{Q}_{\infty}(\Psi, \Psi)=0, t \rightarrow \infty$. Therefore, (1.12) follows from (7.1). Thus, we may assume that for a given $\Psi \in \mathscr{D}^{0}$,

$$
\mathscr{Q}_{\infty}(\Psi, \Psi) \neq 0
$$

Let us choose $0<\delta<1$ and

$$
\rho_{t} \sim t^{1-\delta}, \quad \Delta_{t} \sim \frac{t}{\log t}, \quad t \rightarrow \infty
$$


Lemma 9.3. The following limit holds true:

$$
N_{t}\left(\varphi\left(\rho_{t}\right)+\left(\frac{\rho_{t}}{t}\right)^{1 / 2}\right)+N_{t}^{2}\left(\varphi^{1 / 2}\left(\rho_{t}\right)+\frac{\rho_{t}}{t}\right) \rightarrow 0, \quad t \rightarrow \infty
$$

Proof. Function $\varphi(r)$ is non-increasing, hence by (2.14),

$$
r^{d} \varphi^{1 / 2}(r)=d \int_{0}^{r} s^{d-1} \varphi^{1 / 2}(r) d s \leqslant d \int_{0}^{r} s^{d-1} \varphi^{1 / 2}(s) d s \leqslant C \bar{\varphi}<\infty .
$$

Furthermore, (9.22) implies that $h_{t}=\rho_{t}+\Delta_{t} \sim \frac{t}{\log t}, t \rightarrow \infty$. Therefore, $N_{t} \sim \frac{t}{h_{t}} \sim \log t$. Then (9.23) follows by (9.24) and (9.22).

By the triangle inequality,

$$
\begin{aligned}
\left|E \exp \left\{i\left\langle Y_{0}, \Phi(\cdot, t)\right\rangle\right\}-\hat{\mu}_{\infty}(\Psi)\right| \\
\leqslant\left|E \exp \left\{i\left\langle Y_{0}, \Phi(\cdot, t)\right\rangle\right\}-E \exp \left\{i \sum_{t} r_{t}^{j}\right\}\right| \\
+\left|\exp \left\{-\frac{1}{2} \sum_{t} E\left|r_{t}^{j}\right|^{2}\right\}-\exp \left\{-\frac{1}{2} \mathscr{Q}_{\infty}(\Psi, \Psi)\right\}\right| \\
+\left|E \exp \left\{i \sum_{t} r_{t}^{j}\right\}-\exp \left\{-\frac{1}{2} \sum_{t} E\left|r_{t}^{j}\right|^{2}\right\}\right| \\
\equiv I_{1}+I_{2}+I_{3} .
\end{aligned}
$$

We are going to show that all summands $I_{1}, I_{2}, I_{3}$ tend to zero as $t \rightarrow \infty$.

Step (i). Equation (9.14) implies

$$
\begin{aligned}
I_{1} & =\left|E \exp \left\{i \sum_{t} r_{t}^{j}\right\}\left(\exp \left\{i \sum_{t} c_{t}^{j}+i l_{t}\right\}-1\right)\right| \\
& \leqslant \sum_{t} E\left|c_{t}^{j}\right|+E\left|l_{t}\right| \leqslant \sum_{t}\left(E\left|c_{t}^{j}\right|^{2}\right)^{1 / 2}+\left(E\left|l_{t}\right|^{2}\right)^{1 / 2} .
\end{aligned}
$$

From (9.26), (9.16), (9.17), and (9.23) we obtain that

$$
I_{1} \leqslant C_{p} t^{-p}+C N_{t}\left(\rho_{t} / t\right)^{1 / 2} \rightarrow 0, \quad t \rightarrow \infty .
$$


Step (ii). By the triangle inequality,

$$
\begin{aligned}
I_{2} \leqslant & \left.\frac{1}{2}\left|\sum_{t} E\right| r_{t}^{j}\right|^{2}-\mathscr{Q}_{\infty}(\Psi, \Psi) \mid \\
\leqslant & \frac{1}{2}\left|\mathscr{Q}_{t}(\Psi, \Psi)-\mathscr{Q}_{\infty}(\Psi, \Psi)\right| \\
& \quad+\left.\frac{1}{2}\left|E\left(\sum_{t} r_{t}^{j}\right)^{2}-\sum_{t} E\right| r_{t}^{j}\right|^{2}\left|+\frac{1}{2}\right| E\left(\sum_{t} r_{t}^{j}\right)^{2}-\mathscr{Q}_{t}(\Psi, \Psi) \mid \\
\equiv & I_{21}+I_{22}+I_{23},
\end{aligned}
$$

where $\mathscr{Q}_{t}$ is a quadratic form with the matrix kernel $\left(Q_{t}^{i j}(x, y)\right)$. (7.1) implies that $I_{21} \rightarrow 0$. As to $I_{22}$, we first have that

$$
I_{22} \leqslant \sum_{j<l}\left|E r_{t}^{j} r_{t}^{l}\right|
$$

The next lemma is a corollary of Lemma 17.2.3 in ref. 17.

Lemma 9.4. Let $\mathscr{A}, \mathscr{B}$ be the subsets of $\mathbb{Z}^{d}$ with the distance $\operatorname{dist}(\mathscr{A}, \mathscr{B}) \geqslant r>0$, and $\xi, \eta$ be random variables on the probability space $\left(\mathscr{H}_{\alpha}, \mathscr{B}\left(\mathscr{H}_{\alpha}\right), \mu_{0}\right)$. Moreover, let $\xi$ be measurable with respect to the $\sigma$-algebra $\sigma(\mathscr{A}), \eta$ with respect to the $\sigma$-algebra $\sigma(\mathscr{B})$. Then

(i) $|E \xi \eta-E \xi E \eta| \leqslant C a b \varphi^{1 / 2}(r)$ if $\left(E|\xi|^{2}\right)^{1 / 2} \leqslant a$ and $\left(E|\eta|^{2}\right)^{1 / 2} \leqslant b$.

(ii) $|E \xi \eta-E \xi E \eta| \leqslant C a b \varphi(r)$ if $|\xi| \leqslant a$ and $|\eta| \leqslant b$ a.e.

We apply Lemma 9.4 to deduce that $I_{22} \rightarrow 0$ as $t \rightarrow \infty$. Note that $r_{t}^{j}=\left\langle Y_{0}, \chi_{t}^{j} \Phi(\cdot, t)\right\rangle$ is measurable with respect to the $\sigma$-algebra $\sigma\left(R_{t}^{j}\right)$. The distance between the different rooms $R_{t}^{j}$ is greater or equal to $\rho_{t}$ according to (9.10). Then (9.29) and S1, S3 imply, together with Lemma 9.4(i) and (9.15), that

$$
I_{22} \leqslant C N_{t}^{2} \varphi^{1 / 2}\left(\rho_{t}\right)
$$

which vanishes as $t \rightarrow \infty$ because of (9.23). Finally, it remains to check that $I_{23} \rightarrow 0, t \rightarrow \infty$. We have

$$
\mathscr{Q}_{t}(\Psi, \Psi)=E\left\langle Y_{0}, \Phi(\cdot, t)\right\rangle^{2}=E\left(\sum_{t}\left(r_{t}^{j}+c_{t}^{j}\right)+l_{t}\right)^{2},
$$


according to (9.14). Therefore, by the Cauchy-Schwartz inequality,

$$
\begin{aligned}
I_{23} & \leqslant\left|E\left(\sum_{t} r_{t}^{j}\right)^{2}-E\left(\sum_{t} r_{t}^{j}+\sum_{t} c_{t}^{j}+l_{t}\right)^{2}\right| \\
& \leqslant C N_{t} \sum_{t} E\left|c_{t}^{j}\right|^{2}+C_{1}\left(E\left(\sum_{t} r_{t}^{j}\right)^{2}\right)^{1 / 2}\left(N_{t} \sum_{t} E\left|c_{t}^{j}\right|^{2}+E\left|l_{t}\right|^{2}\right)^{1 / 2}+C E\left|l_{t}\right|^{2}
\end{aligned}
$$

Then (9.15), (9.29), and (9.30) imply

$$
E\left(\sum_{t} r_{t}^{j}\right)^{2} \leqslant \sum_{t} E\left|r_{t}^{j}\right|^{2}+2 \sum_{j<l}\left|E r_{t}^{j} r_{t}^{l}\right| \leqslant C N_{t} \Delta_{t} / t+C_{1} N_{t} \varphi^{1 / 2}\left(\rho_{t}\right) \leqslant C_{2}<\infty
$$

Now (9.16), (9.17), (9.31), and (9.23) yield

$$
I_{23} \leqslant C_{1} N_{t}^{2} \rho_{t} / t+C_{2} N_{t}\left(\rho_{t} / t\right)^{1 / 2}+C_{3} t^{-p} \rightarrow 0, \quad t \rightarrow \infty .
$$

So, all terms $I_{21}, I_{22}, I_{23}$ in (9.28) tend to zero. Then (9.28) implies that

$$
I_{2} \leqslant\left.\frac{1}{2}\left|\sum_{t} E\right| r_{t}^{j}\right|^{2}-\mathscr{Q}_{\infty}(\Psi, \Psi) \mid \rightarrow 0, \quad t \rightarrow \infty
$$

Step (iii). It remains to verify that

$$
I_{3}=\left|E \exp \left\{i \sum_{t} r_{t}^{j}\right\}-\exp \left\{-\frac{1}{2} \sum_{t} E\left|r_{t}^{j}\right|^{2}\right\}\right| \rightarrow 0, \quad t \rightarrow \infty .
$$

Lemma 9.4(ii) yields:

$$
\begin{aligned}
\mid E \exp & \left\{i \sum_{t} r_{t}^{j}\right\}-\prod_{-N_{t}}^{N_{t}-1} E \exp \left\{i r_{t}^{j}\right\} \mid \\
\leqslant & \left|E \exp \left\{i r_{t}^{-N_{t}}\right\} \exp \left\{i \sum_{-N_{t}+1}^{N_{t}-1} r_{t}^{j}\right\}-E \exp \left\{i r_{t}^{-N_{t}}\right\} E \exp \left\{i \sum_{-N_{t}+1}^{N_{t}-1} r_{t}^{j}\right\}\right| \\
& +\left|E \exp \left\{i r_{t}^{-N_{t}}\right\} E \exp \left\{i \sum_{-N_{t}+1}^{N_{t}-1} r_{t}^{j}\right\}-\prod_{-N_{t}}^{N_{t}-1} E \exp \left\{i r_{t}^{j}\right\}\right| \\
\leqslant & C \varphi\left(\rho_{t}\right)+\left|E \exp \left\{i \sum_{-N_{t}+1}^{N_{t}-1} r_{t}^{j}\right\}-\prod_{-N_{t}+1}^{N_{t}-1} E \exp \left\{i r_{t}^{j}\right\}\right| .
\end{aligned}
$$


We then apply Lemma 9.4(ii) recursively and get, according to Lemma 9.3,

$$
\left|E \exp \left\{i \sum_{t} r_{t}^{j}\right\}-\prod_{-N_{t}}^{N_{t}-1} E \exp \left\{i r_{t}^{j}\right\}\right| \leqslant C N_{t} \varphi\left(\rho_{t}\right) \rightarrow 0, \quad t \rightarrow \infty .
$$

It remains to check that

$$
\left|\prod_{-N_{t}}^{N_{t}-1} E \exp \left\{i r_{t}^{j}\right\}-\exp \left\{-\frac{1}{2} \sum_{t} E\left|r_{t}^{j}\right|^{2}\right\}\right| \rightarrow 0, \quad t \rightarrow \infty .
$$

According to the standard statement of the Central Limit Theorem (see, e.g., ref. 21, Theorem 4.7), it suffices to verify the Lindeberg condition: $\forall \delta>0$,

$$
\frac{1}{\sigma_{t}} \sum_{t} E_{\delta \sqrt{\sigma_{t}}}\left|r_{t}^{j}\right|^{2} \rightarrow 0, \quad t \rightarrow \infty
$$

Here $\sigma_{t} \equiv \sum_{t} E\left|r_{t}^{j}\right|^{2}$, and $E_{\varepsilon} f \equiv E\left(X_{\varepsilon} f\right)$, where $X_{a}$ is the indicator of the event $|f|>\varepsilon^{2}$. Note that (9.33) and (9.21) imply that $\sigma_{t} \rightarrow \mathscr{Q}_{\infty}(\Psi, \Psi) \neq 0$, $t \rightarrow \infty$. Hence it remains to verify that

$$
\sum_{t} E_{\varepsilon}\left|r_{t}^{j}\right|^{2} \rightarrow 0, \quad t \rightarrow \infty, \quad \text { for any } \quad \varepsilon>0
$$

We check Eq. (9.34) in Section 10. This will complete the proof of Proposition 2.11 .

\section{THE LINDEBERG CONDITION}

The proof of (9.34) can be reduced to the case when for some $\Lambda \geqslant 0$ we have that

$$
\left|u_{0}(x)\right|+\left|v_{0}(x)\right| \leqslant \Lambda<\infty, \quad x \in \mathbb{Z}^{d} .
$$

Then the proof of (9.34) is reduced to the convergence

$$
\sum_{t} E\left|r_{t}^{j}\right|^{4} \rightarrow 0, \quad t \rightarrow \infty
$$

by using Chebyshev's inequality. The general case can be covered by standard cutoff arguments by taking into account that the bound (9.15) for $E\left|r_{t}^{j}\right|^{2}$ depends only on $e_{0}$ and $\varphi$. The last fact is obvious from (9.19) and (5.4). We deduce (10.2) from 
Theorem 10.1. Let the conditions of Theorem A hold and assume that (10.1) is fulfilled. Then for any $\Psi \in \mathscr{D}^{0}$ the following bounds hold:

$$
E\left|r_{t}^{j}\right|^{4} \leqslant C(\Psi) \Lambda^{4} \Delta_{t}^{2} / t^{2}, \quad t>1
$$

\section{Proof.}

Step 1. Given four points $x^{1}, x^{2}, x^{3}, x^{4} \in \mathbb{Z}^{d}$, set: $M_{0}^{(4)}\left(x^{1}, \ldots, x^{4}\right)=$ $E\left(Y_{0}\left(x^{1}\right) \otimes \cdots \otimes Y_{0}\left(x^{4}\right)\right)$. Then, similarly to (9.18), Eqs. (10.1) and (9.13) imply

$$
E\left|r_{t}^{j}\right|^{4}=\left\langle\chi_{t}^{j}\left(x^{1}\right) \cdots \chi_{t}^{j}\left(x^{4}\right) M_{0}^{(4)}\left(x^{1}, \ldots, x^{4}\right), \Phi\left(x^{1}, t\right) \otimes \cdots \otimes \Phi\left(x^{4}, t\right)\right\rangle .
$$

Let us analyze the domain of the $\left(\mathbb{Z}^{d}\right)^{4}$ in the r.h.s. of (10.4). We partition $\left(\mathbb{Z}^{d}\right)^{4}$ into three parts, $W_{2}, W_{3}$, and $W_{4}$ :

$$
\left(\mathbb{Z}^{d}\right)^{4}=\bigcup_{i=2}^{4} W_{i}, W_{i}=\left\{\bar{x}=\left(x^{1}, x^{2}, x^{3}, x^{4}\right) \in\left(\mathbb{Z}^{d}\right)^{4}:\left|x^{1}-x^{i}\right|=\max _{p=2,3,4}\left|x^{1}-x^{p}\right|\right\} \text {. }
$$

Furthermore, given $\bar{x}=\left(x^{1}, x^{2}, x^{3}, x^{4}\right) \in W_{i}$, divide $\mathbb{Z}^{d}$ into three parts $S_{j}$, $j=1,2,3: \mathbb{Z}^{d}=S_{1} \cup S_{2} \cup S_{3}$, by two hyperplanes orthogonal to the segment $\left[x^{1}, x^{i}\right]$ and partitioning it into three equal segments, where $x^{1} \in S_{1}$ and $x^{i} \in S_{3}$. Denote by $x^{p}, x^{q}$ the two remaining points with $p, q \neq 1, i$. Set: $\mathscr{A}_{i}=\left\{\bar{x} \in W_{i}: x^{p} \in S_{1}, x^{q} \in S_{3}\right\}, \mathscr{B}_{i}=\left\{\bar{x} \in W_{i}: x^{p}, x^{q} \notin S_{1}\right\}$, and $\mathscr{C}_{i}=\left\{\bar{x} \in W_{i}: x^{p}, x^{q} \notin S_{3}\right\}, i=2,3,4$. Then $W_{i}=\mathscr{A}_{i} \cup \mathscr{B}_{i} \cup \mathscr{C}_{i}$. Define the function $\mathrm{m}_{0}^{(4)}(\bar{x}), \bar{x} \in\left(\mathbb{Z}^{d}\right)^{4}$, in the following way:

$$
\left.\mathrm{m}_{0}^{(4)}(\bar{x})\right|_{W_{i}}=\left\{\begin{array}{l}
M_{0}^{(4)}(\bar{x})-Q_{0}\left(x^{1}, x^{p}\right) \otimes Q_{0}\left(x^{i}, x^{q}\right), \quad \bar{x} \in \mathscr{A}_{i}, \\
M_{0}^{(4)}(\bar{x}), \quad \bar{x} \in \mathscr{B}_{i} \cup \mathscr{C}_{i} .
\end{array}\right.
$$

This determines $\mathrm{m}_{0}^{(4)}(\bar{x})$ correctly for all quadruples $\bar{x}$. Note that

$$
\begin{aligned}
\left\langle\chi_{t}^{j}\left(x^{1}\right)\right. & \left.\cdots \chi_{t}^{j}\left(x^{4}\right) Q_{0}\left(x^{1}, x^{p}\right) \otimes Q_{0}\left(x^{i}, x^{q}\right), \Phi\left(x^{1}, t\right) \otimes \cdots \otimes \Phi\left(x^{4}, t\right)\right\rangle \\
= & \left\langle\chi_{t}^{j}\left(x^{1}\right) \chi_{t}^{j}\left(x^{p}\right) Q_{0}\left(x^{1}, x^{p}\right), \Phi\left(x^{1}, t\right) \otimes \Phi\left(x^{p}, t\right)\right\rangle \\
& \times\left\langle\chi_{t}^{j}\left(x^{i}\right) \chi_{t}^{j}\left(x^{q}\right) Q_{0}\left(x^{i}, x^{q}\right), \Phi\left(x^{i}, t\right) \otimes \Phi\left(x^{q}, t\right)\right\rangle .
\end{aligned}
$$

Each factor here is bounded by $C(\Psi) \Delta_{t} / t$. Similarly to $(9.15)$, this can be deduced from an expression of type (9.18) for the factors. Therefore, the proof of (10.3) reduces to the proof of the bound

$$
\begin{aligned}
I_{t} & :=\left|\left\langle\chi_{t}^{j}\left(x^{1}\right) \cdots \chi_{t}^{j}\left(x^{4}\right) \mathrm{m}_{0}^{(4)}\left(x^{1}, \ldots, x^{4}\right), \Phi\left(x^{1}, t\right) \otimes \cdots \otimes \Phi\left(x^{4}, t\right)\right\rangle\right| \\
& \leqslant C(\Psi) \Lambda^{4} \Delta_{t}^{2} / t^{2}, \quad t>1 .
\end{aligned}
$$


Step 2. Similarly to (9.19), the estimate (9.6) implies,

$$
I_{t} \leqslant C t^{-2 d} \sum_{\bar{x}} \chi_{t}^{j}\left(x^{1}\right) \cdots \chi_{t}^{j}\left(x^{4}\right)\left|\mathrm{m}_{0}^{(4)}\left(x^{1}, \ldots, x^{4}\right)\right| .
$$

We estimate $\mathrm{m}_{0}^{(4)}$ using Lemma 9.4(ii).

Lemma 10.2. For each $i=2,3,4$ and all $\bar{x} \in W_{i}$ the following bound holds:

$$
\left|\mathrm{m}_{0}^{(4)}\left(x^{1}, \ldots, x^{4}\right)\right| \leqslant C \Lambda^{4} \varphi\left(\left|x^{1}-x^{i}\right| / 3\right) .
$$

Proof. For $\bar{x} \in \mathscr{A}_{i}$ we apply Lemma 9.4(ii) to $\mathbb{R}^{2 n} \otimes \mathbb{R}^{2 n}$-valued random variables $\xi=Y_{0}\left(x^{1}\right) \otimes Y_{0}\left(x^{p}\right)$ and $\eta=Y_{0}\left(x^{i}\right) \otimes Y_{0}\left(x^{q}\right)$. Then (10.1) implies the bound for all $\bar{x} \in \mathscr{A}_{i}$,

$$
\left|\mathrm{m}_{0}^{(4)}(\bar{x})\right| \leqslant C \Lambda^{4} \varphi\left(\left|x^{1}-x^{i}\right| / 3\right) .
$$

For $\bar{x} \in \mathscr{B}_{i}$, we apply Lemma 9.4(ii) to $\xi=Y_{0}\left(x_{1}\right)$ and $\eta=Y_{0}\left(x^{p}\right) \otimes Y_{0}\left(x^{q}\right)$ $\otimes Y_{0}\left(x^{i}\right)$. Then $\mathrm{S} 0$ implies a similar bound for all $\bar{x} \in \mathscr{B}_{i}$,

$$
\begin{aligned}
\left|\mathrm{m}_{0}^{(4)}(\bar{x})\right| & =\left|M_{0}^{(4)}(\bar{x})-E Y_{0}\left(x^{1}\right) \otimes E\left(Y_{0}\left(x_{p}\right) \otimes Y_{0}\left(x^{q}\right) \otimes Y_{0}\left(x^{i}\right)\right)\right| \\
& \leqslant C \Lambda^{4} \varphi\left(\left|x^{1}-x^{i}\right| / 3\right),
\end{aligned}
$$

and the same for all $\bar{x} \in \mathscr{C}_{i}$.

Step 3. It remains to prove the following bounds for each $i=2,3,4$ :

$$
V_{i}(t):=\sum_{\bar{x}} \chi_{t}^{j}\left(x^{1}\right) \cdots \chi_{t}^{j}\left(x^{4}\right) X_{i}(\bar{x}) \varphi\left(\left|x^{1}-x^{i}\right| / 3\right) \leqslant C \Delta_{t}^{2} t^{2 d-2},
$$

where $X_{i}$ is an indicator of the set $W_{i}$. In fact, this sum does not depend on $i$, hence set $i=2$ in the summand:

$$
V_{i}(t) \leqslant C \sum_{x^{1}, x^{2}} \chi_{t}^{j}\left(x^{1}\right) \chi_{t}^{j}\left(x^{2}\right) \varphi\left(\left|x^{1}-x^{2}\right| / 3\right) \sum_{x^{3}} \chi_{t}^{j}\left(x^{3}\right) \sum_{x^{4}} \chi_{t}^{j}\left(x^{4}\right) X_{2}(\bar{x}) .
$$

Now a key observation is that the inner sum in $x^{4}$ is $\mathcal{O}\left(\left|x^{1}-x^{2}\right|^{d}\right)$ as $X_{2}(\bar{x})=0$ for $\left|x^{4}-x^{1}\right|>\left|x^{1}-x^{2}\right|$. This implies

$$
V_{i}(t) \leqslant C \sum_{x^{1}} \chi_{t}^{j}\left(x^{1}\right) \sum_{x^{2}} \chi_{t}^{j}\left(x^{2}\right) \varphi\left(\left|x^{1}-x^{2}\right| / 3\right)\left|x^{1}-x^{2}\right|^{d} \sum_{x^{3}} \chi_{t}^{j}\left(x^{3}\right) .
$$


Remember that $\chi_{t}^{j}(x)$ is an indicator of the room $R_{t}^{j}=\left\{x \in \mathbb{Z}^{d}:|x| \leqslant N_{t} h_{t}\right.$, $\left.a^{j} \leqslant x_{d}<b^{j}\right\}$, where $N_{t}=\left[\gamma t / h_{t}\right]$. The inner sum in $x^{2}$ is bounded as

$$
\begin{aligned}
& \int_{\left|x^{2}\right| \leqslant \gamma t} \varphi\left(\left|x^{1}-x^{2}\right| / 3\right)\left|x^{1}-x^{2}\right|^{d} d x^{2} \\
& \leqslant C(d) \int_{0}^{2 \gamma t} r^{2 d-1} \varphi(r / 3) d r \\
& \leqslant C_{1}(d) \sup _{r \in[0,2 \gamma t]} r^{d} \varphi^{1 / 2}(r / 3) \int_{0}^{2 \gamma t} r^{d-1} \varphi^{1 / 2}(r / 3) d r,
\end{aligned}
$$

where the "sup" and the last integral are bounded by (9.24) and (2.14), respectively. Therefore, (10.12) follows from (10.14). This completes the proof of Theorem 10.1.

\section{APPENDIX A. DYNAMICS AND COVARIANCE IN FOURIER SPACE}

Proof of Proposition 2.4. Applying Fourier transform to (1.3) we obtain

$$
\dot{\hat{Y}}(t)=\hat{\mathscr{A}}(\theta) \hat{Y}(t), \quad t \in \mathbb{R}, \quad \hat{Y}(0)=\hat{Y}_{0} .
$$

Here we denote

$$
\hat{\mathscr{A}}(\theta)=\left(\begin{array}{cc}
0 & 1 \\
-\hat{V}(\theta) & 0
\end{array}\right), \quad \theta \in T^{d} .
$$

Note that $\hat{Y}(\cdot, t) \in D^{\prime}\left(T^{d}\right)$ for $t \in \mathbb{R}$. On the other hand, $\hat{V}(\theta)$ is a smooth function by E1. Therefore, the solution $\hat{Y}(\theta, t)$ of (A.1) exists, is unique and admits the representation $\hat{Y}(\theta, t)=\exp (\hat{\mathscr{A}}(\theta) t) \hat{Y}_{0}(\theta)$ which becomes the convolution

$$
Y(x, t)=\sum_{x^{\prime} \in \mathbb{Z}^{d}} \mathscr{G}_{t}\left(x-x^{\prime}\right) Y_{0}\left(x^{\prime}\right)
$$

in the coordinate space, where the Green function $\mathscr{G}_{t}(z)$ admits the Fourier representation

$$
\mathscr{G}_{t}(z):=F_{\theta \rightarrow z}^{-1}[\exp (\hat{\mathscr{A}}(\theta) t)]=(2 \pi)^{-d} \int_{T^{d}} e^{-i z \theta} \exp (\hat{\mathscr{A}}(\theta) t) d \theta
$$

Hence, by the partial integration, $\mathscr{G}_{t}(z) \sim|z|^{-p}$ as $|z| \rightarrow \infty$ for any $p>0$ and bounded $|t|$ since $\hat{\mathscr{A}}(\theta)$ is the smooth function of $\theta \in T^{d}$. Therefore, the convolution representation (A.3) implies $Y(t) \in \mathscr{H}_{\alpha}$. 
Covariance in Fourier Space. Note that $\hat{\mathscr{G}}_{t}(\theta)$ has a form

$$
\hat{\mathscr{G}}_{t}(\theta)=\left(\begin{array}{cc}
\cos \Omega t & \sin \Omega t \Omega^{-1} \\
-\sin \Omega t \Omega & \cos \Omega t
\end{array}\right),
$$

where $\Omega=\Omega(\theta)$ is the Hermitian matrix defined by (2.2). Let $\hat{C}(\theta)$ be defined by (2.21) and $I$ be the identity matrix. Then

$$
\hat{\mathscr{G}}_{t}(\theta)=\cos \Omega t I+\sin \Omega t \hat{C}(\theta) .
$$

Denote by $Q(x, y):=E\left(Y_{0}(x) \otimes Y_{0}(y)\right), \quad$ and $\quad Q_{t}(x, y):=E(Y(x, t) \otimes$ $Y(y, t))$. Hence, applying Fourier transform to $Q_{t}(x, y)$ we get

$$
\hat{Q}_{t}\left(\theta, \theta^{\prime}\right):=F_{x \rightarrow \theta, y \rightarrow-\theta^{\prime}} Q_{t}(x, y)=\hat{\mathscr{G}}_{t}(\theta) \hat{Q}\left(\theta, \theta^{\prime}\right) \hat{\mathscr{G}}_{t}^{T}\left(-\theta^{\prime}\right),
$$

where $\hat{Q}\left(\theta, \theta^{\prime}\right):=F_{x \rightarrow \theta, y \rightarrow-\theta^{\prime}} Q(x, y)$. Note that due to condition E2 $\Omega^{T}\left(-\theta^{\prime}\right)=\Omega^{*}\left(\theta^{\prime}\right)=\Omega\left(\theta^{\prime}\right)$ and then $\hat{\mathscr{G}}_{t}^{T}\left(-\theta^{\prime}\right)=\hat{\mathscr{G}}_{t}^{*}\left(\theta^{\prime}\right)$, where

$$
\hat{\mathscr{G}}_{t}^{*}(\theta):=\cos \Omega t I+\sin \Omega t \hat{C}^{*}(\theta) .
$$

Here $\hat{C}^{*}$ is Hermitian adjoint matrix to $\hat{C}$ as in (2.21). Then

$$
\begin{aligned}
\hat{Q}_{t}\left(\theta, \theta^{\prime}\right)= & \cos \Omega(\theta) t \hat{Q}\left(\theta, \theta^{\prime}\right) \cos \Omega\left(\theta^{\prime}\right) t \\
& +\sin \Omega(\theta) t \hat{C}(\theta) \hat{Q}\left(\theta, \theta^{\prime}\right) \hat{C}^{*}\left(\theta^{\prime}\right) \sin \Omega\left(\theta^{\prime}\right) t \\
& +\cos \Omega(\theta) t \hat{Q}\left(\theta, \theta^{\prime}\right) \hat{C}^{*}\left(\theta^{\prime}\right) \sin \Omega\left(\theta^{\prime}\right) t \\
& +\sin \Omega(\theta) t \hat{C}(\theta) \hat{Q}\left(\theta, \theta^{\prime}\right) \cos \Omega\left(\theta^{\prime}\right) t
\end{aligned}
$$

Now, for simplicity of calculations, we will assume that the set of the "crossing" points $\theta_{*}$ is empty, i.e., $\omega_{k}(\theta) \neq \omega_{l}(\theta), \forall k, l \in \bar{n}$, and the functions $\omega_{k}(\theta)$ and $B(\theta)$ are real-analytic. For example, this is the case of the simple elastic lattice (3.1). (Otherwise, we need a partition of unity (8.7)). Consider the first term in the r.h.s. of (A.8). We rewrite it using (2.3) in the form

$$
\begin{aligned}
\cos \Omega(\theta) t \hat{Q}\left(\theta, \theta^{\prime}\right) \cos \Omega\left(\theta^{\prime}\right) t \\
=B(\theta)\left(\cos \omega_{k}(\theta) t A\left(\theta, \theta^{\prime}\right)_{k l} \cos \omega_{l}\left(\theta^{\prime}\right) t\right)_{k, l \in \bar{n}} B^{*}\left(\theta^{\prime}\right) \\
=B(\theta) \frac{1}{2}\left(\left(\cos \left(\omega_{k}(\theta)-\omega_{l}\left(\theta^{\prime}\right)\right) t\right.\right. \\
\left.\left.\quad+\cos \left(\omega_{k}(\theta)+\omega_{l}\left(\theta^{\prime}\right)\right) t\right) A\left(\theta, \theta^{\prime}\right)_{k l}\right)_{k, l \in \bar{n}} B^{*}\left(\theta^{\prime}\right),
\end{aligned}
$$


where $A\left(\theta, \theta^{\prime}\right):=B^{*}(\theta) \hat{Q}\left(\theta, \theta^{\prime}\right) B\left(\theta^{\prime}\right)$. Similarly, we can rewrite the remaining three terms in the r.h.s. of (A.8). Finally,

$$
\hat{Q}_{t}\left(\theta, \theta^{\prime}\right)=\hat{\mathscr{G}}_{t}(\theta) \hat{Q}\left(\theta, \theta^{\prime}\right) \hat{\mathscr{G}}_{t}^{*}\left(\theta^{\prime}\right)=B(\theta) R_{t}\left(\theta, \theta^{\prime}\right) B^{*}\left(\theta^{\prime}\right),
$$

where by $R_{t}\left(\theta, \theta^{\prime}\right)$ we denote the $2 n \times 2 n$ matrix with the entries

$$
\begin{aligned}
R_{t}\left(\theta, \theta^{\prime}\right)_{k l}:= & \frac{1}{2} \sum_{ \pm}\left\{\cos \left(\omega_{k}(\theta) \pm \omega_{l}\left(\theta^{\prime}\right)\right) t\right. \\
& \times\left[B^{*}(\theta)\left(\hat{Q}\left(\theta, \theta^{\prime}\right) \mp \hat{C}(\theta) \hat{Q}\left(\theta, \theta^{\prime}\right) \hat{C}^{*}\left(\theta^{\prime}\right)\right) B\left(\theta^{\prime}\right)\right]_{k l} \\
& +\sin \left(\omega_{k}(\theta) \pm \omega_{l}\left(\theta^{\prime}\right)\right) t \\
& \left.\times\left[B^{*}(\theta)\left(\hat{C}(\theta) \hat{Q}\left(\theta, \theta^{\prime}\right) \pm \hat{Q}\left(\theta, \theta^{\prime}\right) \hat{C}^{*}\left(\theta^{\prime}\right)\right) B\left(\theta^{\prime}\right)\right]_{k l}\right\}
\end{aligned}
$$

In the translation-invariant case $Q(x, y)=q(x-y), \hat{Q}\left(\theta, \theta^{\prime}\right)=\delta\left(\theta-\theta^{\prime}\right)$ $\times \hat{q}(\theta)$ and we get

$$
\hat{Q}_{t}\left(\theta, \theta^{\prime}\right)=\delta\left(\theta-\theta^{\prime}\right) B(\theta) R_{t}(\theta) B^{*}(\theta),
$$

where by $R_{t}(\theta)$ we denote the $2 n \times 2 n$ matrix with the entries

$$
\begin{aligned}
R_{t}(\theta)_{k l}= & \frac{1}{2} \sum_{ \pm}\left\{\cos \left(\omega_{k}(\theta) \pm \omega_{l}(\theta)\right) t\left[B^{*}(\theta)\left(\hat{q}(\theta) \mp \hat{C}(\theta) \hat{q}(\theta) \hat{C}^{*}(\theta)\right) B(\theta)\right]_{k l}\right. \\
& \left.+\sin \left(\omega_{k}(\theta) \pm \omega_{l}(\theta)\right) t\left[B^{*}(\theta)\left(\hat{C}(\theta) \hat{q}(\theta) \pm \hat{q}(\theta) \hat{C}^{*}(\theta)\right) B(\theta)\right]_{k l}\right\}
\end{aligned}
$$

Let us denote by $p(\theta):=B^{*}(\theta) \hat{q}(\theta) B(\theta)$. Then by (2.3) and (2.21) we obtain

$$
\begin{aligned}
R_{t}(\theta)_{k l}= & \frac{1}{2} \sum_{ \pm}\left\{\cos \left(\omega_{k}(\theta) \mp \omega_{l}(\theta)\right) t\left(\begin{array}{cc}
p_{k l}^{00} \pm \omega_{k}^{-1} p_{k l}^{11} \omega_{l}^{-1} & p_{k l}^{01} \mp \omega_{k}^{-1} p_{k l}^{10} \omega_{l} \\
p_{k l}^{10} \mp \omega_{k} p_{k l}^{01} \omega_{l}^{-1} & p_{k l}^{11} \pm \omega_{k} p_{k l}^{00} \omega_{l}
\end{array}\right)\right. \\
& \left.+\sin \left(\omega_{k}(\theta) \pm \omega_{l}(\theta)\right) t\left(\begin{array}{cc}
\omega_{k}^{-1} p_{k l}^{10} \pm p_{k l}^{01} \omega_{l}^{-1} & \omega_{k}^{-1} p_{k l}^{11} \mp p_{k l}^{00} \omega_{l} \\
-\omega_{k} p_{k l}^{00} \pm p_{k l}^{11} \omega_{l}^{-1} & -\omega_{k} p_{k l}^{01} \mp p_{k l}^{10} \omega_{l}
\end{array}\right)\right\}
\end{aligned}
$$

We enumerate the eigenvalues $\omega_{k}(\theta)$ as in (2.5). Then for $k, l \in\left(r_{\sigma-1}, r_{\sigma}\right]$, $\sigma=1, \ldots, s+1$, we have

$$
\begin{aligned}
R_{t}(\theta)_{k l}= & \frac{1}{2}\left[B^{*}(\theta)\left(\hat{q}(\theta)+\hat{C}(\theta) \hat{q}(\theta) \hat{C}^{*}(\theta)\right) B(\theta)\right]_{k l} \\
& +\frac{1}{2} \cos 2 \omega_{k}(\theta) t\left[B^{*}(\theta)\left(\hat{q}(\theta)-\hat{C}(\theta) \hat{q}(\theta) \hat{C}^{*}(\theta)\right) B(\theta)\right]_{k l} \\
& +\frac{1}{2} \sin 2 \omega_{k}(\theta) t\left[B^{*}(\theta)\left(\hat{C}(\theta) \hat{q}(\theta)+\hat{q}(\theta) \hat{C}^{*}(\theta)\right) B(\theta)\right]_{k l}
\end{aligned}
$$




\section{ACKNOWLEDGMENTS}

T.V.D. and A.I.K. were supported partly by research grants of DFG (436 RUS 113/615/0-1), RFBR (99-01-04012), and the FWF, Projects Y 137-TEC and P16105-N05. A.I.K. was supported partly by Max-Planck Institute for Mathematics in the Sciences (Leipzig). N.J.M. was supported partly by the START project "Nonlinear Schrödinger and Quantum Boltzmann Equations” (FWF Y 137-TEC).

\section{REFERENCES}

1. C. Boldrighini, A. Pellegrinotti, and L. Triolo, Convergence to stationary states for infinite harmonic systems, J. Stat. Phys. 30:123-155 (1983).

2. F. Bonetto, J. L. Lebowitz, and L. Rey-Bellet, Fourier law: A challenge to theorists, in Mathematical Physics 2000, A. Fokas et al., eds. (Imperial College Press, London, 2000), pp. 128-150, arXiv: math-ph/0002052.

3. I. P. Cornfeld, S. V. Fomin, and Ya. G. Sinai, Ergodic Theory (Springer, New York, 1981).

4. R. L. Dobrushin and Yu. M. Suhov, On the problem of the mathematical foundation of the Gibbs postulate in classical statistical mechanics, in Mathematical Problems in Theoretical Physics, Lecture Notes in Physics, Vol. 80 (Springer-Verlag, Berlin, 1978), pp. $325-340$.

5. T. V. Dudnikova and A. I. Komech, Ergodic properties of hyperbolic equations with mixing, Theory Probab. Appl. 41:436-448 (1996).

6. T. V. Dudnikova, Stabilization of space-time statistical solutions of the Klein-Gordon equation, Russian J. Math. Phys. 5:176-188 (1997).

7. T. V. Dudnikova, A. I. Komech, E. A. Kopylova, and Yu. M. Suhov, On convergence to equilibrium distribution, I. The Klein-Gordon equation with mixing, Commun. Math. Phys. 225:1-32 (2002).

8. T. V. Dudnikova, A. I. Komech, N. E. Ratanov, and Yu. M. Suhov, On convergence to equilibrium distribution, II. The wave equation in odd dimensions, with mixing, J. Stat. Phys. 108:1219-1253 (2002).

9. T. V. Dudnikova, A. I. Komech, and H. Spohn, On a two-temperature problem for wave equation, Markov Process. Related Fields 8:43-80 (2002).

10. T. Dudnikova, A. Komech, and H. Spohn, On convergence to statistical equilibrium for harmonic crystal, accepted to J. Math. Phys. (2003), ArXiv: math-ph/0210039.

11. J.-P. Eckmann, C.-A. Pillet, and L. Rey-Bellet, Non-equilibrium statistical mechanics of anharmonic chains coupled to two heat baths at different temperatures, Commun. Math. Phys. 201:657-697 (1999).

12. J.-P. Eckmann, C.-A. Pillet, and L. Rey-Bellet, Entropy production in nonlinear, thermally driven Hamiltonian systems, J. Stat. Phys. 95:305-331 (1999).

13. M. V. Fedoryuk, The stationary phase method and pseudodifferential operators, Russian Math. Surveys 26:65-115 (1971).

14. J. Farmer, S. Goldstein, and E. R. Speer, Invariant states of a thermally conducting barrier, J. Stat. Phys. 34:263-277 (1984).

15. F. Fidaleo and C. Liverani, Ergodic properties for a quantum nonlinear dynamics, J. Stat. Phys. 97:957-1009 (1999).

16. V. Jakšić and C.-A. Pillet, Ergodic properties of classical dissipative systems. I, Acta Math. 181:245-282 (1998). 
17. I. A. Ibragimov and Yu. V. Linnik, Independent and Stationary Sequences of Random Variables (Wolters-Noordhoff, Groningen, 1971).

18. E. A. Kopylova, Stabilization of statistical solutions of the Klein-Gordon equation, Moscow Univ. Math. Bull. 41:72-75 (1986).

19. O. E. Lanford, III and J. L. Lebowitz, Time evolution and ergodic properties of harmonic systems, in Dynamical Systems, Theory, and Applications, Lecture Notes in Physics, Vol. 38 (Springer-Verlag, Berlin, 1975).

20. H. Nakazawa, On the lattice thermal conduction, Progr. Theoret. Phys. (Suppl.) 45:231-262 (1970).

21. V. V. Petrov, Limit Theorems of Probability Theory (Clarendon Press, Oxford, 1995).

22. M. Reed and B. Simon, Methods of Modern Mathematical Physics III: Scattering Theory (Academic Press, New York, 1979).

23. L. Rey-Bellet and L. E. Thomas, Exponential convergence to non-equilibrium stationary states in classical statistical mechanics, Commun. Math. Phys. 225:305-329 (2002).

24. Z. Rieder, J. L. Lebowitz, and E. Lieb, Properties of a harmonic crystal in a stationary nonequilibrium state, J. Math. Phys. 8:1073-1078 (1967).

25. M. A. Rosenblatt, A central limit theorem and a strong mixing condition, Proc. Nat. Acad. Sci. U.S.A. 42:43-47 (1956).

26. A. G. Shuhov and Yu. M. Suhov, Ergodic properties of groups of the Bogoliubov transformations of CAR $C^{*}$-algebras, Ann. Physics 175:231-266 (1987).

27. H. Spohn and J. Lebowitz, Stationary non-equilibrium states of infinite harmonic systems, Comm. Math. Phys. 54:97-120 (1977).

28. M. I. Vishik and A. V. Fursikov, Mathematical Problems of Statistical Hydromechanics (Kluwer Academic, Dordrecht, 1988). 